\title{
Combining randomized and non-randomized evidence in network meta-analysis
}

\begin{tabular}{|c|c|}
\hline Journal: & Statistics in Medicine \\
\hline Manuscript ID & SIM-16-0419.R2 \\
\hline Wiley - Manuscript type: & Research Article \\
\hline Date Submitted by the Author: & 06-Dec-2016 \\
\hline Complete List of Authors: & $\begin{array}{l}\text { Efthimiou, Orestis; University of Ioannina School of Medicine, Department } \\
\text { of Hygiene and Epidemiology } \\
\text { Mavridis, Dimitris; University of Ioannina School of Medicine, Department } \\
\text { of Hygiene and Epidemiology; University of Ioannina, Department of } \\
\text { Primary Education } \\
\text { Debray, Thomas; University Medical Center Utrecht, Julius Center for } \\
\text { Health Sciences and Primary Care; University Medical Center Utrecht, The } \\
\text { Dutch Cochrane Centre, Julius Center for Health Sciences and Primary Care } \\
\text { Samara, Myrto; Technische Universität München, Department of Psychiatry } \\
\text { and Psychotherapy } \\
\text { Belger, Mark; Eli Lilly and Company, Lilly Research Centre } \\
\text { Siontis, George; Bern University Hospital, Department of Cardiology } \\
\text { Leucht, Stefan; Technische Universität München, Department of Psychiatry } \\
\text { and Psychotherapy } \\
\text { Salanti, Georgia; University of Ioannina School of Medicine, Department of } \\
\text { Hygiene and Epidemiology; University of Bern, Institute of Social and } \\
\text { Preventive Medicine; University of Bern, Berner Institut für } \\
\text { Hausarztmedizin (BIHAM) }\end{array}$ \\
\hline Keywords: & $\begin{array}{l}\text { observational studies, observational evidence, observational data, multiple } \\
\text { treatments meta-analysis, mixed treatment comparison }\end{array}$ \\
\hline
\end{tabular}




\title{
Combining randomized and non-randomized evidence in network meta-analysis
}

\author{
Orestis Efthimiou $^{1}$, Dimitris Mavridis ${ }^{1,2}$, Thomas P. A. Debray ${ }^{3,4}$, M. Samara ${ }^{5}$, M. Belger ${ }^{6}$, George \\ C.M. Siontis ${ }^{7}$, S. Leucht ${ }^{5}$ and Georgia Salanti ${ }^{1,8,9}$ on behalf of GetReal Work Package 4
}

\begin{abstract}
Non-randomized studies aim to reveal whether or not interventions are effective in real-life clinical practice and there is a growing interest in including such evidence in the decision-making process. We evaluate existing methodologies and present new approaches to using non-randomized evidence in a network meta-analysis (NMA) of randomized controlled trials (RCTs) when the aim is to assess relative treatment effects. We first discuss how to assess compatibility between the two types of evidence. We then present and compare an array of alternative methods that allow the inclusion of non-randomized studies in an NMA of RCTs: the naïve data synthesis, the designadjusted synthesis, the use of non-randomized evidence as prior information and the use of threelevel hierarchical models. We apply some of the methods in two previously published clinical examples comparing percutaneous interventions for the treatment of coronary in-stent restenosis and antipsychotics in patients with schizophrenia. We discuss in depth the advantages and limitations of each method and we conclude that the inclusion of real-world evidence from nonrandomized studies has the potential to corroborate findings from RCTs, increase precision and enhance the decision-making process.
\end{abstract}

Keywords: observational studies; observational evidence; observational data; multiple treatments meta-analysis; mixed treatment comparison; cohort studies.

\section{Introduction}

Pairwise and network meta-analyses (NMAs) are often limited to synthesizing evidence from randomized controlled trials (RCTs). NMAs frequently disregard evidence from non-randomized studies (NRSs) because the authors assume estimates of relative treatment effects are more likely to

\footnotetext{
${ }^{1}$ Department of Hygiene and Epidemiology, University of Ioannina School of Medicine, Ioannina, Greece

${ }^{2}$ Department of Primary Education, University of Ioannina, Ioannina, Greece

${ }^{3}$ Julius Center for Health Sciences and Primary Care, University Medical Center Utrecht, Utrecht, The Netherlands

${ }^{4}$ Cochrane Netherlands, Julius Center for Health Sciences and Primary Care, University Medical Center Utrecht, Utrecht, The Netherlands

${ }^{5}$ Department of Psychiatry and Psychotherapy, Technische Universität München, München, Germany

${ }^{6}$ Eli Lilly and Company, Lilly Research Centre, Windlesham, UK

${ }^{7}$ Department of Cardiology, Bern University Hospital, Bern, Switzerland

${ }^{8}$ Institute of Social and Preventive Medicine, University of Bern, Bern, Switzerland

${ }^{9}$ Berner Institut für Hausarztmedizin (BIHAM), University of Bern, Bern, Switzerland
} 
be biased, especially when confounding has been inadequately addressed. When non-randomized evidence is included in an NMA, this amplifies concerns about transitivity and consistency assumed by the method, and fears that results may be very precise, yet biased. But interest in including NRSs in the NMA synthesis and decision-making process is growing [1-5]. Although RCTs are considered the most reliable source of information on relative treatment effects, their strictly experimental setting and inclusion criteria may limit their ability to predict results in real-world clinical practice [6]. NRS-based estimates of treatment effects may complement evidence provided by RCTs, and potentially address some of their limitations. However, less than $4 \%$ of the NMAs published until the end of 2012 included at least one non-randomized study (10 out of 261 identified NMAs) [7].

Expert opinion is required to formulate quantitative statements about the amount of bias propagated by non-randomized studies in a body of evidence [8]. A recent review summarized methods that account for potential bias from non-randomized evidence in a pairwise meta-analysis [9]. These include an additive bias model that accounts for both external and internal biases in studies [10]; the confidence profile method [11]; likelihood adjustments [12]; and, multiple-bias models [13]. Schmitz et al. [14] propose three different methods to combine data from different study designs in an NMA: 'naïve' pooling; use of non-randomized evidence as prior information; and, a three-level hierarchical model.

In this paper we present statistical methods for combining randomized and non-randomized evidence in an NMA and we discuss their merits and limitations. We confine ourselves to the case where NMA is used to infer about the relative effects of health interventions. To this end, we consider only comparative non-randomized studies that aim to estimate relative treatment effects. These include observational studies as well as comparative clinical trials that do not employ randomization.

\section{Description of the motivating examples}

\subsection{Percutaneous interventions for the treatment of coronary in-stent restenosis}

A previously published NMA synthesized aggregate data from 28 published RCTs (5914 patients) that compared 8 different percutaneous interventional strategies for the treatment of coronary in-stent restenosis [15]. The follow-up in the included studies ranged from 6 to 60 months after the indexed intervention and several clinical outcomes were considered; we focus on the dichotomous outcome 'target-lesion revascularization' (TLR). Results were synthesized using the odds-ratio (OR).

In addition to the RCTs, we identified data from 6 NRSs that provide evidence about TLR on 5 interventions. The studies included a total of 1113 patients in 14 different cohorts. The network is 
depicted in panel A, Figure 1. Detailed information about the included NRSs can be found in Section 4 of the Appendix.

\subsection{Antipsychotic drugs in schizophrenia}

The randomized evidence in this example consists of aggregate per-arm data from 167 RCTs (36871 patients) which compared 15 antipsychotic drugs and placebo in patients diagnosed with schizophrenia [16]. Change in symptoms (efficacy) was measured 4-12 weeks after randomization, based on the brief psychiatric rating scale (BPRS) or the positive and negative syndrome scale (PANSS). We use the standardized mean difference (SMD) to synthesize data. Using SMD as the effect measure enables the meta-analysis of studies that employ different scales, and it was also used in the original analysis [16]; researchers should note, however, that the use of SMD may be problematic under circumstances [17]. Study-level information was available for participant's mean age and duration of illness.

Non-randomized evidence consists of IPD from a large observational study (SOHO, Schizophrenia Outpatient Health Outcome) with 8873 adult patients from 10 European countries, who were treated with five different antipsychotics during a 3-year time frame [18]. Short-term change in symptoms was measured at three months, based on the Clinical Global Impression scale (CGI). The network is depicted in panel B, Figure 1. Because we have signed non-disclosure agreements with our industry partner who provided the observational data we use in this example, we code treatments as 1-16.

\section{[INSERT FIGURE 1]}

\section{Statistical methods}

We present the methods assuming that relative treatments from non-randomized evidence have been estimated using valid epidemiological and statistical methods that aim to minimize bias if possible. An overview of such methods can be found in Faria et al. [3]. When considering the inclusion of NRS for which IPD are not available, extra caution is warranted as the aggregated reported effect estimates may originate from suboptimal analyses. In any case, the quality of the evidence provided by the identified NRSs needs to be critically appraised. The recently proposed ROBINS-I tool can be used to evaluate the risk of bias in estimates obtained from studies that did not use randomization [19]. If the identified NRSs are believed to have a very high risk of selection bias their inclusion in NMA would be difficult to defend.

None of the NRSs about the effects of percutaneous interventions for coronary in-stent restenosis provided IPD. To estimate the SMDs for the antipsychotics from IPD in SOHO we use regression adjustment analysis since there was enough overlap between the distributions of patient characteristics across treatment groups [3]. After consulting with expert psychiatrists to indicate 
important patient-level covariates the SMDs from the non-randomized data were adjusted for baseline severity of the illness, age, gender and duration of illness.

In Section 3.1 we discuss the standard NMA model and fix notation. In Section 3.1, we describe methods for identifying possible discrepancies between randomized and non-randomized evidence. In Section 3.3, we present methods for synthesizing the two sources of evidence, assuming that no important differences have been found between them. We also describe the similarities and differences between the methods. Additional details for all models can be found in the Appendix.

\subsection{Standard NMA model with aggregated data}

The usual hierarchical, random-effects (RE) NMA model [20, 21] synthesizes data from all available studies to estimate the summary treatment effect, $\mu_{X Y}$, and the heterogeneity standard deviation of the random effects, $\tau_{X Y}$, for each treatment comparison $\mathrm{X}$ vs. Y. Assume that for a twoarm XY study $j$ we observe the relative treatment effect $d_{j X Y}$, along with a standard error, $s_{j X Y}$. The model is then written as:

$$
\begin{gathered}
d_{j X Y} \sim N\left(\theta_{j X Y}, s_{j X Y}^{2}\right) \\
\theta_{j X Y} \sim N\left(\mu_{X Y}, \tau_{X Y}^{2}\right)
\end{gathered}
$$

We assume consistency, i.e. $\mu_{X Y}=\mu_{X Z}-\mu_{Y Z}$ for any choice of treatments $\mathrm{X}, \mathrm{Y}$ and $\mathrm{Z}$. This reduces the number of parameters and sets it equal to the number of treatments in the network minus one. Choosing treatment $\mathrm{A}$ as the reference, it is sufficient to estimate $\mu_{A X}$ for all treatments $X \neq A$; these are called the basic parameters. All other treatment effects can be obtained as linear combinations of the estimated $\mu_{A X}$. Treatments can be ranked by any measure that summarizes the overlap between the estimated distributions of $\mu_{A X}$ [22]. An assumption commonly employed to facilitate the estimation of the heterogeneity parameters is to assume $\tau_{X Y}=\tau$ for all treatments $\mathrm{X}$ and $\mathrm{Y}$ [23]. We use this assumption throughout this article for simplicity, although it is not necessary for any of the methods discussed below. For the inclusion of multi-arm studies the model described above is expanded to allow for both within and between-study correlations of the observations by using multivariate distributions. For a comprehensive review of standard NMA methodology, we refer the reader to our recent publication [24].

\subsection{Assessing the agreement within and across randomized and non-randomized evidence}

An NMA of RCTs should be internally consistent: information from direct and indirect sources of evidence for each treatment comparison should agree. The presence of inconsistency can be tested statistically [25] and any disagreements could be explored in subgroup analysis or network meta-regression. In the presence of large unexplained inconsistency NMA may be inappropriate. If no substantial inconsistencies are found in the NMA of RCTs one can proceed with the evaluation of the agreement between randomized and non-randomized evidence. Disagreements between 
randomized and non-randomized evidence might be due to confounding in the non-randomized evidence, important differences in treatment effect modifiers across treatment groups of the NRSs, systematic differences in the definition of treatments between experimental and real-world settings (e.g. differences in dosages, modes of administration, adherence, etc.) or differences in patient characteristics. Inclusion criteria in RCTs are usually strict, so patients included in randomized studies may systematically differ from patients included in studies of real-world clinical settings.

Examination of differences between direct and indirect evidence can be formalised by graphical and statistical comparison of the following information for each pairwise comparison XY:

i. direct randomized evidence, from XY randomized trials;

ii. indirect randomized evidence for $\mathrm{XY}$, from the network, after excluding all direct $\mathrm{XY}$ studies;

iii. direct non-randomized evidence, from NRSs that include X and Y treatment arms.

iv. indirect, non-randomized evidence.

The four sources of evidence are independent and they can be formally compared with statistical tests. The tests for inconsistency are however low-powered and the usefulness of formal statistical inference will be limited [26]. Note that differences between (i) and (ii) correspond to the notion of inconsistency in NMA [27].

Another, informal way to infer about agreement between randomized and non-randomized studies is to compare the estimated heterogeneity parameters between the two different datasets. If NRSs are very different from RCTs, their inclusion into the network shall lead to an important increase in the heterogeneity parameter.

If researchers identify a source of disagreement between randomized and non-randomized evidence they can perform analyses that account for it and improve comparability across the different sources of evidence. Cooper et al. [28] and Salanti et al. [29] presented a general network meta-regression framework for including study-level covariates in an NMA. We discuss network meta-regression models in more detail in Section 1 of the Appendix. Concerns about limited power and ecological bias are just as relevant in network meta-regression as in conventional metaregression. Additionally, using network meta-regression in practice might prove to be difficult or even completely infeasible, e.g. if there is no usable information on important study-level covariates. Other models beyond network meta-regression might be useful in addressing specific sources of heterogeneity and inconsistency. Differences in the definitions of the treatments could be explicitly modelled using previously presented approaches $[30,31]$. When there are differences in the way that the outcomes are measured or reported (e.g. at different time points or using different scales), multiple outcomes NMA could be employed [32-34]. 
Researchers should note that even in the case of agreement between randomized and nonrandomized evidence it may be inappropriate to perform a joint NMA. Epidemiological assessment of the compatibility of the various sources of data should always be performed by a content expert before undertaking any form of joint synthesis. The identified NRSs need to be examined on whether or not they are sufficiently similar in terms of population, intervention, comparator and outcomes (PICO) with the ones specified in the research question that the review is aiming to answer [2]. When a NRS is deemed to be incompatible with the specific aims of the research question it should be excluded from all analyses, irrespective of whether its findings happen to agree with those from the RCTs.

\subsection{Methods for combining randomized and non-randomized evidence}

If the NMA of RCTs is consistent and provided that there is no evidence of substantial disagreement between the randomized and the (adjusted) non-randomized evidence, synthesis of data is warranted. Below we outline different approaches to synthesis and summarize their basic characteristics in Table 1. We distinguish three main methodological approaches: (A) synthesis of non-randomized and randomized studies where information from NRSs is manipulated to reflect confidence in the study findings; $(B)$ constructing informative prior distribution from the nonrandomized evidence and subsequently use it in the NMA of RCTs; $(C)$ a three-level hierarchical model, where one level of the model accounts for differences in RCT and NRS designs.

Before embarking on these adjusted analyses investigators can obtain an initial insight of the effect of including NRSs in the network by synthesizing all data from all studies ('naïve' analysis) [14].

\section{A. The 'Design-adjusted' evidence synthesis}

This approach synthesizes randomized and non-randomized studies, after adjusting the mean effect sizes and/or the variance of the latter. In a two-armed non-randomized study $j$ the point estimate is shifted by $\beta_{j}$, where $\beta_{j}$ is a bias term. The variance of the mean effect is also inflated (divided by a factor $w_{j}$, with $0<w_{j}<1$ ), so that the study's weight in the meta-analysis decreases. The investigator must define not only values for each set of $\beta_{j}$ and but also the direction of bias; that is which treatment is assumed to be 'favored'. The standard NMA model of Equation (1) is modified as follows:

$$
\begin{gathered}
d_{j X Y} \sim N\left(\theta_{j X Y}+\beta_{j}, \frac{s_{j X Y}^{2}}{w_{j}}\right) \\
\theta_{j X Y} \sim N\left(\mu_{X Y}, \tau_{X Y}^{2}\right)
\end{gathered}
$$

For a multi-armed study $j$ with $T_{j}$ treatment groups a bias vector $\boldsymbol{\beta}_{\boldsymbol{j}}$ with $T_{j}-1$ elements needs to be specified, along with $w_{j}$ to inflate the within-study variance-covariance matrix of the 
observations. Estimation of heterogeneity variances can be performed as usual in NMA, e.g. using likelihood methods, the methods of moments or within a Bayesian framework. The parameters $\beta_{j}$ or $w_{j}$ can be set equal to fixed values or can be treated as random variables within a Bayesian framework. A Bayesian approach offers maximum flexibility and would allow us to assign prior distributions to $\beta_{j}$ or $w_{j}$. Note that if a prior distribution is assigned to $\beta_{j}$ then there is no need to do any additional down-weighting using $w_{j}$, since the additional uncertainty incorporated in $\beta_{j}$ will result to a down-weighting of study $j$. These prior distributions can be specified to reflect potential deficiencies in the non-randomized studies. For example, we may assign a prior for $w_{j}$ centered to values above 0.5 or even close to one to a well-conducted NRS with low or moderate risk of bias and PICO characteristics that are relevant to the research question. In contrast, we could assign a distribution for $w_{j}$ that is centered at low values below 0.5 or close to 0 to a NRS of high risk of bias or a NRS that does not quite address the research question. Alternatively, $\beta_{j}$ or $w_{j}$ can be treated as random variables exchangeable across the included NRSs [35]. In the presence of discrepancies between NRSs and RCTs and if $w_{j}$ are treated as random variables, their posterior will be centered around smaller values limiting the effect of the NRSs [36].

Even though NRSs are generally considered to be at higher risk of bias [1], it may be hard to predict the direction (and also the magnitude) of possible biases in treatment effects [37-41], and this, in turn, makes it hard to pinpoint values for $\beta_{j}$ and $w_{j}$. Biases in estimates of relative effects from NRSs may also depend on the method used to obtain them. Different methods used to estimate relative treatment effects from a NRS could give different results, making it harder to quantify potential biases. We recommend that researchers vary the amount of confidence they place on the non-randomized evidence by varying the value of $w_{j}$, and thus gauge the effect of non-randomized evidence on the model estimates. Setting all $w_{j} \approx 0$ corresponds to an NMA of only RCTs. Setting larger values $w_{j}$ places more confidence in non-randomized estimates. Setting all $w_{j}=1$ is equivalent to the naïve model described above, where results from the NRSs are taken at face value. We do not recommend using values $w_{j}>1$, which place more confidence on the NRSs.

Eliciting expert opinion methods is needed to provide a range of plausible values for $\beta_{j}$ and $w_{j}$. Turner et al. [8] discuss how to elicit expert opinion regarding the bias parameters, and how to pool information from different experts. We previously described another method that can be used to pool expert opinion for a parameter ranging from 0 to 1 (such as $w_{j}$ ) which is based on beta distributions [32]. In this method each expert opinion can be weighted according to the expert's experience in the field. An approach alternative to expert opinion is to use information from 
previously published meta-epidemiological studies (e.g. Anglemyer et al. [41]) using a model such as the one presented by Welton et al. [42].

\section{B. Using non-randomized evidence as prior information}

Most NMAs with RCTs are done within a Bayesian framework [7], and non-informative priors are typically assumed for all basic parameters $\mu_{A X}$. In the presence of non-randomized evidence, however, these priors could be informative. The analysis is performed in two steps. First, a metaanalysis (or an NMA, if possible) uses the non-randomized evidence to estimate mean relative treatment effects for some (or all) of the basic parameters. The (posterior) distributions estimated in this first step can be 'adjusted' for bias (by adding a bias parameter $\beta$ to the mean and dividing the variance by $w$ ). Then these distributions are used in a second step as prior distributions for (some, or all of) the basic parameters of the NMA model which includes only RCTs. There are three different ways to construct informative priors. Expert opinion is needed for each one of them, for setting the values needed to adjust NRS.

The first approach is to start by synthesizing non-randomized evidence to estimate $\hat{\mu}_{A X}^{N R S}$ with a corresponding variance $\widehat{V}_{A X}^{N R S}$. This estimated variance $\widehat{V}_{A X}^{N R S}$ incorporates both the sampling error and heterogeneity, so that the distribution $N\left(\hat{\mu}_{A X}^{N R S}, \widehat{V}_{A X}^{N R S}\right)$ corresponds to the so-called 'predictive distribution' [43]. Then, down-weighting of the non-randomized evidence is achieved by assuming $\mu_{A X} \sim N\left(\hat{\mu}_{A X}^{N R S}+\beta_{A X}, \widehat{V}_{A X}^{N R S} / w_{A X}\right)$, where $\beta_{A X}$ is a comparison-specific bias parameter and $0<w_{A X}<1$ is an inflation factor that quantifies the confidence to be placed in the nonrandomized evidence for $A X$ [14]. The model can be extended to incorporate uncertainty in these two parameters by assigning an informative prior distribution to $\beta_{A X}$ or to $w_{A X}$ (but not both). When the $w$ parameters are assumed to be random, their posterior distribution depends on the agreement between the sources of evidence. When randomized and non-randomized evidence disagree, $w$ will obtain smaller values and decrease the impact of the non-randomized evidence on the pooled estimates. Setting all $\beta_{A X}=0$ and $w_{A X}=1$ corresponds to placing full confidence in the nonrandomized evidence; in such a case, and under the fixed-effect (FE) assumption, approach B becomes equivalent to a FE naïve analysis. Note that if some of the NRSs are multi-armed the corresponding estimates on basic parameters will be correlated. In such cases, we need to use multivariate prior distributions. For example, an A versus $\mathrm{X}$ versus $\mathrm{Y}$ non-randomized study will provide information on $\mu_{A X}, \mu_{A Y}$ and the corresponding variance-covariance matrix; these can be used to formulate a bivariate normal prior distribution on the two basic parameters.

Another method for constructing the prior is to use the exact likelihood of the non-randomized data. The evidence provided by NRS $j$ can be down-weighted by raising the likelihood to a power $0 \leq a_{j} \leq 1$; this corresponds to the power-prior originally proposed by Ibrahim and Chen [44, 45]. 
Dividing the variance by $w_{j}$ (approach A) can be seen as a special case of the power prior method, where a study-specific power $a_{j}$ is chosen for the likelihood of each study. In the special case of normally distributed data, approach A and the power prior approach are equivalent: $a_{j}$ corresponds to $w_{j}$.

A third alternative approach is to use a mixture of priors $[46,47]$. The prior distribution consists of two parts; an informative prior formed by the predictive distribution as described above, and a flat (uninformative) prior. A factor $0 \leq p \leq 1$ controls the amount of information drawn from the informative part of the prior, and thus controls the contribution of non-randomized evidence to the final results. For the special case when normal distributions are used for both the informative and the uninformative parts of the prior, we can calculate how the mixture parameter $p$ relates to the variance inflation parameter $w$. More details can be found in Section 3 of the Appendix.

The major difference between approaches A and B is the way that heterogeneity is accounted for in the analysis. In approach A, there is a single heterogeneity parameter for the relative treatment effects estimated in RCTs or NRSs. Approach B assumes two different heterogeneity parameters; one for NRSs and one for the RCTs. This is relevant because NRSs may tend to be more dissimilar than RCTs. When few NRSs are available (as in the example of antipsychotics), researchers cannot use a random-effects meta-analysis to formulate a predictive prior distribution, unless they make some assumption about heterogeneity. For example, the estimate from the naïve analysis or empirical data can use used to construct the predictive distributions. One important limitation of using NRSs to formulate prior distributions is that it precludes the option to explore differences with RCTs in a network meta regression model.

\section{Three-level hierarchical models}

Three-level hierarchical models are suitable to synthesize data from studies with many different designs (e.g., different RCT designs, controlled trials, cohort studies, case-control, etc.) [14, 48, 49]. We present three different realizations of a hierarchical model for NMA. The main difference between them is the way the analysis incorporates the consistency assumption. We denote the various study designs with $i$. At the first level, each study is analyzed separately to obtain estimates of the relative effects of the treatments that are compared in the study.

\section{Model C.1}

At the second level in this model, NMA (including the consistency equations) is used to synthesize studies of the same design to obtain design-specific NMA estimates for the basic parameters and the heterogeneity variance $\tau_{i}^{2}$.

$$
d_{i j X Y} \sim N\left(\theta_{i j X Y}, s_{i j X Y}^{2}\right)
$$




$$
\theta_{i j X Y} \sim N\left(\kappa_{i A Y}-\kappa_{i A X}, \tau_{i}^{2}\right)
$$

The model can be used to include multi-arm studies using standard NMA methodology. Although we assume a single heterogeneity parameter within each design for simplicity, the model can be written using comparison-specific heterogeneities. At the third level, the basic parameters are assumed to be exchangeable across designs, which accounts for possible design-level heterogeneity, $\tau_{d e s}^{2}$, i.e. $\kappa_{i A X} \sim N\left(\mu_{A X}, \tau_{d e s}^{2}\right), \kappa_{i A Y} \sim N\left(\mu_{A Y}, \tau_{d e s}^{2}\right)$, etc. For the case of two different study designs (RCTs and NRSs), setting $\tau_{\text {des }}^{2}=0$ renders the model equivalent to approach B (with $w_{X A}=1$ and $\beta_{A X}=0$ for all basic parameters). Note that model C. 1 requires an NMA to be performed for each study design using a subset of the same set of basic parameters. This might be infeasible in practice (see Section 4 of the Appendix for an illustration. Model C.1 cannot be used in such cases).

\section{Model C.2}

At the second level we perform a series of pairwise, design-specific meta-analyses to obtain summary effects for all available treatment comparisons. The meta-analyses do not impose the consistency equations, but assume a common heterogeneity $\tau_{i}^{2}$ for all treatment comparisons within design $i$, which corresponds to the 'unrelated mean effects model' described by Dias et al. [50]:

$$
\begin{gathered}
d_{i j X Y} \sim N\left(\theta_{i j X Y}, s_{i j X Y}^{2}\right) \\
\theta_{i j X Y} \sim N\left(\kappa_{i X Y}, \tau_{i}^{2}\right)
\end{gathered}
$$

If the common heterogeneity assumption is relaxed the model corresponds to a series of unrelated pairwise meta-analyses.

At the third level, a random-effects NMA (with heterogeneity $\tau_{d e s}^{2}$ ) uses the consistency equations to synthesize estimates from the second level, i.e. we assume that $\kappa_{i X Y} \sim N\left(\mu_{A Y}-\right.$ $\left.\mu_{A X}, \tau_{\text {des }}^{2}\right)$. Model C.2 is problematic when the dataset contains multi-arm trials, because different parameterizations of the unrelated mean effects model can result into different estimates $\mu_{i X Y}$ [50].

\section{Model C.3}

At the second level an NMA is performed separately per design. This imposes consistency exactly as in model C.1. Again the heterogeneity within each design can be assumed to be common, or different parameters can be assigned to different comparisons. At the third level, the estimates of the basic-parameters and their variance-covariance matrix from the design-level NMAs are synthesized in a new NMA, where they are treated as multi-arm studies and consistency equations are again imposed. This model assumes consistency twice - once within, and once across designs. At the third level, the common heterogeneity parameter of the NMA is $\tau_{d e s}^{2}$. Note that this model does not require all NMAs at the second level to estimate a subset of the basic parameters. 
In any three-level hierarchical model the estimates from each study can be adjusted if appropriate, by shifting the mean and/or inflating the variance. Alternatively, evidence from each design can be down-weighted by inflating the variance of the estimates obtained at the second level.

A major constraint in applying models C.1, C.2 and C3 is the availability of data from many different designs. Using an informative prior distribution for $\tau_{d e s}^{2}$ will improve estimates in the presence of few designs. This prior distribution could be formulated using expert opinion that takes into account the expected dispersion of results in different designs. Alternatively, information of meta-epidemiological studies that include information on multiple types of designs (e.g. RCTs, registries, pragmatic trials, observational studies etc.) could be used to form empirical prior distributions.

\subsection{Comparison of models}

The three approaches presented here have similarities and, under specific circumstances, can lead to identical or equivalent statistical models. Frequentist and Bayesian implementation of model A with non-informative or weakly informative priors and the (necessarily Bayesian) Model B should give similar yet not identical results. Differences between frequentist and Bayesian methods are likely to result primarily from the estimation of heterogeneity parameters (which is assumed to be a random variable within the Bayesian context), as any prior distribution for these types of parameters tends to be informative. It is also possible to modify the models' characteristics, and to combine their distinctive features. For example, the effect sizes and variances of each NRS can be adjusted separately (as in model A) before using the three-level hierarchical model (C). Table 1 offers guidance for choosing a model appropriate to apply in practice.

\section{[INSERT TABLE 1]}

\section{Estimating the influence of the non-randomized evidence in the results from NMA}

Once the non-randomized evidence is synthesized jointly with the randomized evidence, the credibility of the final summary treatment effects needs to be evaluated. The evaluation should consider, among other, the risk of bias in the included studies. One cannot exclude the possibility of residual bias due to the inclusion of NRSs, even after excluding studies with high risk of bias or adjusting effect estimates for relevant confounders. Although bias adjustments are usually considered only for the case of NRSs, RCTs may also suffer from design deficiencies that may bias their results. Calculating the relative contributions of the various sources of evidence is important when forming a critical appraisal of the NMA results. This is achieved by taking into account the various design limitations as well as how much each design is contributing to the final NMA treatment effect estimates [51]. 
It has been shown that each relative treatment effect estimated in an NMA can be expressed as the weighted average of a set of direct pairwise meta-analyses [52]. The weights of pairwise metaanalyses in estimating each NMA treatment effect form the 'hat matrix' [52], and can also be presented in a contribution matrix [51]. Since each direct meta-analysis is the weighted average of study-specific results, the contribution matrix can be updated to show the percentage contribution of each study in the pooled NMA estimates. A recent application of this approach can be found in [53]. The study contributions can be grouped to present how much evidence the randomized and non-randomized studies finally contribute to the NMA results. The contribution matrix for an NMA can be obtained with available software, like the netmeta command in R [54] or the netweight command in Stata [55].

Contributions can be readily calculated in approach A when the model is fitted in a frequentist setting, and can provide a percentage contribution of each study. A contribution matrix could also be estimated in approach C. In model C. 1 it is possible to calculate the contribution each design makes to each meta-analysis of basic parameters at the third level, and then sum these contributions within each design. For models C.2 and C.3 the contribution matrix of the NMA, which is performed at the third level (the design level), contains information on the effect of each design on the pooled summary effects.

Calculating study contributions in approach B (and generally for models fitted in Bayesian setting) is not straightforward. A measure similar to a multivariate $I^{2}$ statistic introduced by Jackson et al. can be used to estimate contributions $\left(I_{R}^{2}\right.$ in [56]). In an NMA model, the $(T-1)^{t h}$ root of the determinant of the variance-covariance matrix (denoted here as $\gamma$ ) measures the precision of the estimates for the basic parameters. The contribution of the non-randomized evidence can then be approximated by the relative decrease in $\gamma$ when priors with various weights are employed. For example, the model is fitted using uninformative prior, so that $\gamma_{R C T}$ is based only on RCTs, and then $\gamma_{\text {all }}$ is estimated using informative priors based on NRSs. Then, the contribution of NRS can be approximated with $I_{N R S}^{2}=\left(\gamma_{R C T}-\gamma_{a l l}\right) / \gamma_{R C T} . I_{N R S}^{2}$ can also be used in models A and C (or any other similar model), but complications arise because the estimate for heterogeneity may change after NRSs are included and the $I_{N R S}^{2}$ may acquire negative values for these models. Also, it might be resource intensive to use it to calculate the contribution of each individual study.

\section{Illustrative examples}

We used the network of the percutaneous interventions for the treatment of coronary in-stent restenosis to illustrate approach A, and we employed the network command in Stata [57, 58]. The network of antipsychotics for schizophrenia was used to illustrate approach B. The mean age and mean duration of illness of participants are important study-level effect modifiers, and we used 
network meta-regression to include them in the analysis. Since estimating heterogeneity $(\tau)$ in the non-randomized evidence is impossible in this example (there is only one NRS), we used the estimate from the naïve analysis to construct predictive distributions. To exemplify approach $\mathrm{C}$ we used the antipsychotics network, after assuming four different study categories; RCTs at low, moderate and high risk of bias (see Section 6 of the Appendix for more detail), and also the observational study. We then treated the groups as different designs. We used model $\mathrm{C} 1$ presented in Section 3.3 to synthesize the data. We fitted approaches B and C in OpenBUGS version 3.2.2 $[59,60]$ (codes are provided in the Appendix). For all analyses we ran two chains that allowed for 200.000 samples after a 100.000 burn-in period, and checked convergence with the Gelman and Rubin diagnostic. Initial values were automatically generated using the default OpenBUGS procedure.

In order to assess compatibility between the various sources of evidence we first decomposed the randomized evidence into its direct and indirect components, and then compared them with the nonrandomized evidence. We used the network command in Stata, which implements the loopspecific approach [61] and the node-splitting approach [27].

\subsection{Assessing the agreement within and across randomized and non-randomized evidence}

\subsubsection{Percutaneous interventions for coronary in-stent restenosis}

In Figure 2 we present the relative treatment effects estimated from direct, indirect randomized and non-randomized evidence. For each treatment comparison the figure shows the treatment effects from the direct randomized evidence (from RCTs that compare the corresponding treatments), the indirect randomized evidence (from the network of RCTs after excluding direct evidence), the direct non-randomized evidence (from NRSs that compare the corresponding treatments) and the indirect non-randomized evidence (from the network of NRSs after excluding direct non-randomized evidence). Randomized and non-randomized evidence are in reasonable agreement for most treatment comparisons. However, for some comparisons (in particular DvsA, DvsC, EvsC and GvsB) there are considerable discrepancies The discrepancies in EvsC and DvsC are mainly driven by a single EC non-randomized study [62] whose results were very different from those in RCTs examining the same comparison. This might be due to chance or indicate important unaccounted confounding in the NRS and differences in the population, setting and interventions between the NRS and the RCTs.

The heterogeneity standard deviation was estimated to be $\hat{\tau}=0.36$ for the RCTs network and $\hat{\tau}=0.45$ for the naïve analysis. The loop-specific approach did not detect any inconsistencies in the RCTs network or in the combined RCTs and NRSs network. The node-splitting approach showed no inconsistencies in the RCTs network, but found inconsistency in the DvsA comparison in the 
combined RCTs and NRSs NMA. This inconsistency is due to the difference in estimates coming from indirect randomized and direct non-randomized evidence as shown in Figure 2. Detailed results from these analyses are presented in Section 5 of the Appendix.

In a real-life application, researchers would need to further explore these disagreements, scrutinizing the identified studies in order to make a judgment on whether or not it is appropriate to pool them in a single NMA. To further exemplify the methods, we assume that the identified differences are due to chance and we proceed into synthesizing the totality of the evidence in an NMA.

\section{[INSERT FIGURE 2]}

\subsubsection{Antipsychotic drugs in schizophrenia}

Figure 3 presents the estimates for treatment comparisons for which both randomized and nonrandomized evidence is available. We detected some disagreement only for the $4 \mathrm{v} 6$ comparison. The confidence intervals of effects from direct randomized and non-randomized evidence overlap to some extent but there is a discrepancy between the estimates that correspond to indirect randomized evidence and the evidence from the single NRS. This might indicate that the adjustment of the nonrandomized data was insufficient (e.g., due to residual confounding). There is considerable heterogeneity in the five RCTs that make up the direct randomized evidence $\left(I^{2}=71 \%, \hat{\tau}=0.33\right)$, while the prediction interval is rather broad $(-1.18,1.23)$ and includes the estimate from the NRS. In such a case, heterogeneity in the RCTs that compare 4v6 should be fully explored.

Comparing estimates from an NMA of the RCTs only with estimates from the naïve NMA also indicated that treatment effects are in agreement between the different sources. The precision of some treatment comparisons increased when non-randomized evidence were included in the network. For instance, the width of the credible interval was reduced by $27 \%$ for the $1 \mathrm{vs} 15$ comparison. We compared the heterogeneity standard deviation before and after including the nonrandomized study and found no differences: $\hat{\tau}=0.08$ (95\% Cr.I. $(0.04,0.12))$ for the RCTs-only network, and $0.09(0.05,0.12)$ for the naïve RCTs and NRS network. The loop-specific and the node-splitting approach did not provide any strong evidence of inconsistency either before or after adding the NRS to the network of RCTs (results are presented in Section 7.1 of the Appendix). We conclude that we found no evidence of important disagreement between randomized and nonrandomized summary effects and that synthesis of both sources is warranted.

[INSERT FIGURE 3] 


\subsection{Using the design-adjusted analysis (approach A) for the coronary in-stent restenosis example}

We employ approach A where we assume that estimates from each NRS are expected to be unbiased ( $\beta=0$ ), with an uncertainty reflected on w. However, we want to down-weight the NRS employing various scenarios for a common variance-inflation factor $(w)$, for all NRSs.

Figure 4 shows the estimated relative treatment effects for all comparisons informed by both randomized and non-randomized studies for various values of confidence placed on NRSs. For some treatment comparisons (CvsA; DvsA; EvsA; GvsA) the inclusion of the NRSs in the network corroborated the findings of the NMA based on RCTs alone and increased the precision in the estimates. For three comparisons (DvsC; EvsD; GvsD) the inclusion of non-randomized evidence pulled the summary effect towards the non-effect line even for low values of $w$. Such a result might potentially shed doubts on whether or not the difference in the efficacy of the corresponding interventions can be translated into difference of real-world effectiveness, and might have interesting clinical implications. However, these changes in the summary effect and their confidence intervals were not sufficient to change the treatment hierarchy estimated using the surface under the cumulative ranking line (SUCRA) [63] which remained unchanged with the various analysis models. Results can be found in Section 5 of the Appendix.

In this example the NRSs accounted for $16 \%$ of the total sample size in the network. In the naïve analysis the contribution of NRSs was $20.3 \%$. The contribution decreased to $19.0 \%$ for $w=0.8$; $16.5 \%$ for $w=0.5$ and $10.3 \%$ for $w=0.2$.

\section{[INSERT FIGURE 4]}

\subsection{Using non-randomized evidence as prior information (approach B) for the schizophrenia example}

In order to use the non-randomized evidence as prior information, we first needed to choose the basic parameters appropriately (see Section 2 of the Appendix on choosing the basic parameters). The single NRS compares treatments $1,4,5,6$, and 15 and consequently we could choose any of those treatments to be the reference treatment; here we chose treatment 1 . We used the NRS to estimate a multivariate predictive distribution for the basic parameters 1vs4, 1vs5, 1vs6, and 1vs15, and we used the heterogeneity estimated from the naïve analysis $(\hat{\tau}=0.09)$. We used a common variance inflation factor for these basic parameters to explore three different scenarios: $w \sim \operatorname{Unif}(0,0.3)$, which places a low level of confidence in the non-randomized evidence; $w \sim \operatorname{Unif}(0.3,0.7)$, which places a medium level of confidence; and $w \sim \operatorname{Unif}(0.7,1)$, which places a high level. The rest of the basic parameters were drawn from non-informative distributions, $N\left(0,100^{2}\right)$. 
Estimates of the relative treatment effects are presented in Figure 5. For most treatment comparisons (1vs5, 1vs15, 4vs5, 4vs15, 5vs6, 5vs15 and 6vs15) the inclusion of the single NRS in the evidence-base confirmed the findings and increased precision of the estimates. As expected, treatment hierarchy was robust to the various prior options. Estimates of the model parameters and SUCRA values are presented in Section 7 of the Appendix.

For the first scenario, $I_{N R S}^{2}$ was calculated to be $4.7 \%$; for the second scenario, it was $7 \%$; and, for the third, it was $8.5 \%$.

[INSERT FIGURE 5]

\subsection{Using the three-level hierarchical model (model C1) for the schizophrenia example}

We assumed that the design 'high RoB RCTs' and the 'NRS' design have the same heterogeneity parameter denoted as $\tau_{\text {high }}$, and that designs 'low RoB' and 'moderate RoB' pertain to $\tau_{\text {low\&moderate }}$.

We used four different values for $w$ ranging from 0 (NRS excluded from the analysis) up to 1 (no down-weighting of the NRS). In Table 2 we present the model estimates only for the basic parameters which were informed by both randomized and non-randomized sources. Comparing to the results of approach $\mathrm{B}$ the most notable difference is the increased imprecision. This is because model $\mathrm{C}$ incorporates an additional source of variability in the model, i.e. the heterogeneity across designs. Another interesting finding is that NRS had a larger impact on results; for no downweighting $(w=1), I_{N R S}^{2}$ was calculated to be $14 \%$ (for the third scenario of model B in the previous section this was $8.5 \%$ ). This increase of the contribution of NRS was because this study was the only source of information for one of the four available designs in the dataset.

For $w=1$ the heterogeneity standard deviations were estimated $\hat{\tau}_{h i g h}=0.07(0.01,0.12)$ and $\hat{\tau}_{\text {low\&moderate }}=0.06(0.003,0.16)$. The estimate for the design-level heterogeneity was $\hat{\tau}_{\text {des }}=0.07$ $(0.02,0.13)$, indicating relatively small differences across different designs. These estimates did not materially change when we used different weighting schemes for the NRS.

[INSERT TABLE 2]

\section{Discussion}

In this article we discussed approaches for incorporating non-randomized evidence in an NMA of RCTs. These approaches should be employed after researchers have performed a formal assessment of the risk of bias and the applicability of the identified studies. This is needed in order to evaluate whether the inclusion of each study is sufficiently justified. We also argue that before performing a joint analysis of randomized and non-randomized evidence, researchers need to ensure the compatibility of the different pieces of evidence, for each treatment comparison. If studies are 
deemed incompatible a priori (i.e. before comparing effect estimates across study designs), their inclusion in the NMA should not be considered.

We grouped the available methods for combining randomized and non-randomized evidence in an NMA into three categories; the design-adjusted analysis, the use of informative prior distributions and the three-level hierarchical models. We do not recommend using the naïve approach as the main method of analysis, but it can be a useful starting point, and can provide insight about the effect of including non-randomized evidence in the analysis. The naïve approach can also be used to assess compatibility of randomized and non-randomized evidence, via monitoring changes in network heterogeneity and inconsistency before and after inclusion of nonrandomized evidence.

The 'design-adjusted' approach extends the naïve approach by considering the design of the studies. The data from non-randomized studies are 'shifted' and down-weighted based on external opinion about their credibility. We recommend using this approach when resources allow for a separate assessment of bias for each non-randomized study. Using the non-randomized evidence to construct informative prior distributions for the basic parameters of the model is an elegant alternative for including non-randomized evidence in the NMA. A key difference with the designadjusted approach is in the estimation of heterogeneity, which is performed separately for RCTs and NRSs. This approach might be more intuitive for clinicians, since they typically have prior opinions about treatment effects based on their experience with patient follow-up, monitoring, and registries. Hierarchical models are more appropriate when data from studies of several different designs are to be synthesized and account for heterogeneity within and across designs. While the other methods assume that the underlying treatment effects are the same across designs, the three-level hierarchical models assume that the treatment effects are different - but exchangeable - across different types of studies.

In our two illustrative examples we employed the design-adjusted approach (in-stent restenosis) and the prior-based approach (schizophrenia), with various degrees of confidence placed to the NRSs. For the in-stent restenosis network the inclusion of NRSs confirmed the findings of the RCTs-only analysis for most comparisons. For some of the comparisons results were shifted, indicating smaller differences in the outcome between the interventions even when low confidence was placed on the non-randomized evidence. For the schizophrenia example the inclusion of nonrandomized evidence did not materially impact on the conclusions of the analysis. Precision of the relative treatment effect estimates increased only slightly when we incorporated non-randomized evidence in the analysis, because the contribution of the single, although very large, nonrandomized study was small compared to that of 167 RCTs. 
Whatever method researchers choose to employ they should keep in mind that it is difficult to predict the magnitude or direction of possible biases introduced by including NRSs in an NMA. We thus advise them to explore the effect of placing different levels of confidence in the nonrandomized evidence before they draw final conclusions. We also recommend that all results should be evaluated after considering the relative contribution of each source of evidence in the pooled estimates. This might be especially relevant for the case that NRSs are used to connect disconnected parts of the network of RCTs; on such occasions the connecting studies may acquire an unduly large contribution for (some) of the network estimates, even after severe down-weighting.

Funding: This work was supported by the Innovative Medicines Initiative Joint Undertaking under grant agreement No 115546, resources of which are composed of financial contribution from the European Union's Seventh Framework Programme (FP7/2007- 2013) and EFPIA companies' in kind contribution. The research leading to these results was conducted as part of the GetReal consortium. For further information please refer to www.imi-getreal.eu. This paper only reflects the personal views of the stated authors.

Acknowledgments: We would like to thank the two anonymous reviewers for their constructive criticism and insightful comments, which helped us largely improve our paper.

Declaration of Conflicting Interests: The Authors declare that there is no conflict of interest.

\section{References}

1. Reeves BC, Higgins JPT, Ramsay C, Shea B, Tugwell P, Wells GA: An introduction to methodological issues when including non-randomised studies in systematic reviews on the effects of interventions. Research Synthesis Methods 2013, 4:1-11.

2. Schünemann HJ, Tugwell P, Reeves BC, Akl EA, Santesso N, Spencer FA, Shea B, Wells G, Helfand M: Non-randomized studies as a source of complementary, sequential or replacement evidence for randomized controlled trials in systematic reviews on the effects of interventions. Research Synthesis Methods 2013, 4:49-62.

3. Faria R, Hernadez Alava M, Manca A, Wailoo AJ: The use of observational data to inform estimates of treatment effectiveness in technology appraisal: methods for comparative individual patient data. NICE DSU technical support document 17: http://www.nicedsu.org.uk/Observationaldata-TSD(2973296).htm . 
4. Cameron C, Fireman B, Hutton B, Clifford T, Coyle D, Wells G, Dormuth CR, Platt R, Toh S:

Network meta-analysis incorporating randomized controlled trials and non-randomized comparative cohort studies for assessing the safety and effectiveness of medical treatments: challenges and opportunities. Systematic Reviews 2015, 4.

5. Bell H, Wailoo AJ, Hernandez M, Grieve R, Faria R, Gibson L, Grimm S: The use of real world data for the estimation of treatment effects in NICE decision making. NICE DSU technical support document: http://www.nicedsu.org.uk/Real-World-Data-RWD(3026863).htm .

6. Rothwell PM: External validity of randomised controlled trials: "To whom do the results of this trial apply?" The Lancet 2005, 365:82-93.

7. Nikolakopoulou A, Chaimani A, Veroniki AA, Vasiliadis HS, Schmid CH, Salanti G:

Characteristics of networks of interventions: a description of a database of 186 published networks. PLOS ONE 2014, 9:e86754.

8. Turner RM, Spiegelhalter DJ, Smith GCS, Thompson SG: Bias modelling in evidence synthesis. Journal of the Royal Statistical Society: Series A (Statistics in Society) 2009, 172:21-47.

9. Verde PE, Ohmann C: Combining randomized and non-randomized evidence in clinical research: a review of methods and applications. Research Synthesis Methods 2015, 6:45-62.

10. Spiegelhalter DJ, Best NG: Bayesian approaches to multiple sources of evidence and uncertainty in complex cost-effectiveness modelling. Statistics in Medicine 2003, 22:3687-3709.

11. Eddy DM, Shachter R: Meta-Analysis by the Confidence Profile Method. Har/Dskt edition. Boston: Academic Press; 1992.

12. Wolpert RL, Mengersen KL: Adjusted Likelihoods for Synthesizing Empirical Evidence from Studies That Differ in Quality and Design: Effects of Environmental Tobacco Smoke. Statistical Science 2004, 19:450-471.

13. Greenland S: Multiple-bias modelling for analysis of observational data. Journal of the Royal Statistical Society: Series A (Statistics in Society) 2005, 168:267-306.

14. Schmitz S, Adams R, Walsh C: Incorporating data from various trial designs into a mixed treatment comparison model. Statistics in Medicine 2013, 32:2935-2949.

15. Siontis GCM, Stefanini GG, Mavridis D, Siontis KC, Alfonso F, Pérez-Vizcayno MJ, Byrne RA, Kastrati A, Meier B, Salanti G, Jüni P, Windecker S: Percutaneous coronary interventional strategies for treatment of in-stent restenosis: a network meta-analysis. The Lancet 2015, 386:655664.

16. Leucht S, Cipriani A, Spineli L, Mavridis D, Orey D, Richter F, Samara M, Barbui C, Engel RR, Geddes JR, Kissling W, Stapf MP, Lässig B, Salanti G, Davis JM: Comparative efficacy and 
tolerability of 15 antipsychotic drugs in schizophrenia: a multiple-treatments meta-analysis. Lancet 2013, 382:951-962.

17. Chapter 9: Analysing data and undertaking meta-analyses. In: Higgins JPT, Green S (editors), Cochrane Handbook for Systematic Reviews of Interventions Version 5.1.0 (updated March 2011). The Cochrane Collaboration, 2011. Available from www.cochrane-handbook.org. [Available from www.cochrane-handbook.org.]

18. Haro JM, Edgell ET, Jones PB, Alonso J, Gavart S, Gregor KJ, Wright P, Knapp M, SOHO Study Group: The European Schizophrenia Outpatient Health Outcomes (SOHO) study: rationale, methods and recruitment. Acta Psychiatrica Scandinavica 2003, 107:222-232.

19. Sterne JA, Hernán MA, Reeves BC, Savović J, Berkman ND, Viswanathan M, Henry D, Altman DG, Ansari MT, Boutron I, Carpenter JR, Chan A-W, Churchill R, Deeks JJ, Hróbjartsson A, Kirkham J, Jüni P, Loke YK, Pigott TD, Ramsay CR, Regidor D, Rothstein HR, Sandhu L, Santaguida PL, Schünemann HJ, Shea B, Shrier I, Tugwell P, Turner L, Valentine JC, et al.: ROBINS-I: a tool for assessing risk of bias in non-randomised studies of interventions. BMJ 2016, 355:i4919.

20. Salanti G, Higgins JP, Ades AE, Ioannidis JP: Evaluation of networks of randomized trials. Statistical Methods in Medical Research 2008, 17:279-301.

21. Dias S, Sutton AJ, Ades AE, Welton NJ: Evidence Synthesis for Decision Making 2 A Generalized Linear Modeling Framework for Pairwise and Network Meta-analysis of Randomized Controlled Trials. Med Decis Making 2013, 33:607-617.

22. Nikolakopoulou A, Mavridis D, Salanti G: Planning future studies based on the precision of network meta-analysis results. Statistics in Medicine 2016, 35:978-1000.

23. Higgins JPT, Whitehead A: Borrowing Strength from External Trials in a Meta-Analysis. Statistics in Medicine 1996, 15:2733-2749.

24. Efthimiou O, Debray TPA, van Valkenhoef G, Trelle S, Panayidou K, Moons KGM, Reitsma JB, Shang A, Salanti G, on behalf of GetReal Methods Review Group: GetReal in network metaanalysis: a review of the methodology. Research Synthesis Methods 2016, 7:236-263.

25. Lu G, Ades AE: Assessing evidence inconsistency in mixed treatment comparisons. Journal of the American Statistical Association 2006, 101:447-459.

26. Veroniki AA, Mavridis D, Higgins JP, Salanti G: Characteristics of a loop of evidence that affect detection and estimation of inconsistency: a simulation study. BMC Medical Research Methodology 2014, 14:106.

27. Dias S, Welton NJ, Caldwell DM, Ades AE: Checking consistency in mixed treatment comparison meta-analysis. Statistics in Medicine 2010, 29:932-944. 
28. Cooper NJ, Sutton AJ, Morris D, Ades AE, Welton NJ: Addressing between-study heterogeneity and inconsistency in mixed treatment comparisons: Application to stroke prevention treatments in individuals with non-rheumatic atrial fibrillation. Statistics in Medicine 2009, 28:1861-1881.

29. Salanti G, Marinho V, Higgins JPT: A case study of multiple-treatments meta-analysis demonstrates that covariates should be considered. Journal of Clinical Epidemiology 2009, 62:857864.

30. Del Giovane C, Vacchi L, Mavridis D, Filippini G, Salanti G: Network meta-analysis models to account for variability in treatment definitions: application to dose effects. Statistics in Medicine 2013, 32:25-39.

31. Warren FC, Abrams KR, Sutton AJ: Hierarchical network meta-analysis models to address sparsity of events and differing treatment classifications with regard to adverse outcomes. Statistics in medicine 2014, 33:2449-2466.

32. Efthimiou O, Mavridis D, Cipriani A, Leucht S, Bagos P, Salanti G: An approach for modelling multiple correlated outcomes in a network of interventions using odds ratios. Statistics in medicine 2014, 33:2275-2287.

33. Efthimiou O, Mavridis D, Riley RD, Cipriani A, Salanti G: Joint synthesis of multiple correlated outcomes in networks of interventions. Biostatistics 2015, 16:84-97.

34. Debray TP, Schuit E, Efthimiou O, Reitsma JB, Ioannidis JP, Salanti G, Moons KG, Workpackage on behalf of G: An overview of methods for network meta-analysis using individual participant data: when do benefits arise? Statistical Methods in Medical Research 2016:962280216660741.

35. Dias S, Welton NJ, Marinho VCC, Salanti G, Higgins JPT, Ades AE: Estimation and adjustment of bias in randomized evidence by using mixed treatment comparison meta-analysis. Journal of the Royal Statistical Society Series A (Statistics in Society) 2010, 173:613-629. 36. Neuenschwander B, Branson M, Spiegelhalter DJ: A note on the power prior. Statistics in Medicine 2009, 28:3562-3566.

37. Concato J, Shah N, Horwitz RI: Randomized, controlled trials, observational studies, and the hierarchy of research designs. The New England Journal of Medicine 2000, 342:1887-1892.

38. Benson K, Hartz AJ: A Comparison of Observational Studies and Randomized, Controlled Trials. New England Journal of Medicine 2000, 342:1878-1886.

39. Viswanathan M, Berkman ND, Dryden DM, Hartling L: Assessing Risk of Bias and Confounding in Observational Studies of Interventions or Exposures: Further Development of the 
RTI Item Bank. Rockville (MD): Agency for Healthcare Research and Quality (US); 2013. [AHRQ Methods for Effective Health Care]

40. Valentine JC, Thompson SG: Issues relating to confounding and meta-analysis when including non-randomized studies in systematic reviews on the effects of interventions. Research Synthesis Methods 2013, 4:26-35.

41. Anglemyer A, Horvath HT, Bero L: Healthcare outcomes assessed with observational study designs compared with those assessed in randomized trials. Cochrane Database of Systematic Reviews 2014, 4:MR000034.

42. Welton NJ, Ades AE, Carlin JB, Altman DG, Sterne J a. C: Models for potentially biased evidence in meta-analysis using empirically based priors. Journal of the Royal Statistical Society: Series A (Statistics in Society) 2009, 172:119-136.

43. Spiegelhalter D, Abrams KR, Myles JP: Bayesian Approaches to Clinical Trials and HealthCare Evaluation. Wiley. DOI: 10.1002/0470092602; .

44. Ibrahim JG, Chen M-H: Power Prior Distributions for Regression Models. Statistical Science 2000, 15:46-60.

45. Ibrahim JG, Chen M-H, Gwon Y, Chen F: The power prior: theory and applications. Statistics in Medicine 2015, 34:3724-3749.

46. Schmidli H, Gsteiger S, Roychoudhury S, O’Hagan A, Spiegelhalter D, Neuenschwander B: Robust meta-analytic-predictive priors in clinical trials with historical control information.

Biometrics 2014, 70:1023-1032.

47. Mutsvari T, Tytgat D, Walley R: Addressing potential prior-data conflict when using informative priors in proof-of-concept studies. Pharmaceutical Statistics 2016, 15:28-36.

48. Prevost TC, Abrams KR, Jones DR: Hierarchical models in generalized synthesis of evidence: an example based on studies of breast cancer screening. Statistics in Medicine 2000, 19:3359-3376. 49. Sutton AJ, Abrams KR: Bayesian methods in meta-analysis and evidence synthesis. Statistical Methods in Medical Research 2001, 10:277-303.

50. Dias S, Welton NJ, Sutton AJ, Caldwell DM, Lu G, Ades AE: Evidence Synthesis for Decision Making 4 Inconsistency in Networks of Evidence Based on Randomized Controlled Trials. Medical Decision Making 2013, 33:641-656.

51. Salanti G, Del Giovane C, Chaimani A, Caldwell DM, Higgins JPT: Evaluating the Quality of Evidence from a Network Meta-Analysis. PLoS ONE 2014, 9:e99682.

52. Krahn U, Binder H, König J: A graphical tool for locating inconsistency in network metaanalyses. BMC Medical Research Methodology 2013, 13:35. 
53. Furukawa TA, Miura T, Chaimani A, Leucht S, Cipriani A, Noma H, Mitsuyasu H, Kanba S, Salanti G: Using the contribution matrix to evaluate complex study limitations in a network metaanalysis: a case study of bipolar maintenance pharmacotherapy review. BMC research notes 2016 , 9:218.

54. Rücker G, Schwarzer G, Krahn U, König J: Netmeta: Network Meta-Analysis Using Frequentist Methods. Available at: Http://www.r-Project.org/. 2015.

55. Chaimani A, Higgins JPT, Mavridis D, Spyridonos P, Salanti G: Graphical Tools for Network Meta-Analysis in STATA. PLoS ONE 2013, 8:e76654.

56. Jackson D, White IR, Riley RD: Quantifying the impact of between-study heterogeneity in multivariate meta-analyses. Statistics in Medicine 2012, 31:3805-3820.

57. StataCorp.: Stata Statistical Software: Release 13. College Station, TX: StataCorp LP. 2013. 58. White IR: Network meta-analysis. Stata Journal 2015, 15:951-985.

59. Lunn DJ, Thomas A, Best N, Spiegelhalter D: WinBUGS \&Ndash; A Bayesian Modelling Framework: Concepts, Structure, and Extensibility. Statistics and Computing 2000, 10:325-337. 60. Lunn D, Spiegelhalter D, Thomas A, Best N: The BUGS project: Evolution, critique and future directions. Statistics in Medicine 2009, 28:3049-3067.

61. Bucher HC, Guyatt GH, Griffith LE, Walter SD: The results of direct and indirect treatment comparisons in meta-analysis of randomized controlled trials. Journal of Clinical Epidemiology 1997, 50:683-691.

62. Almalla M, Schröder J, Pross V, Marx N, Hoffmann R: Paclitaxel-eluting balloon versus everolimus-eluting stent for treatment of drug-eluting stent restenosis. Catheterization and Cardiovascular Interventions 2014, 83:881-887.

63. Salanti G, Ades AE, Ioannidis JPA: Graphical methods and numerical summaries for presenting results from multiple-treatment meta-analysis: an overview and tutorial. Journal of clinical epidemiology 2011, 64:163-171. 
Table 1: Overview of the presented approaches for combining randomized and non-randomized evidence. Abbreviations: NMA=network metaanalysis. $\mathrm{RE}=$ Random-effects. $\mathrm{NRS}=$ non-randomized study. $\mathrm{RCT}=$ randomized controlled trial

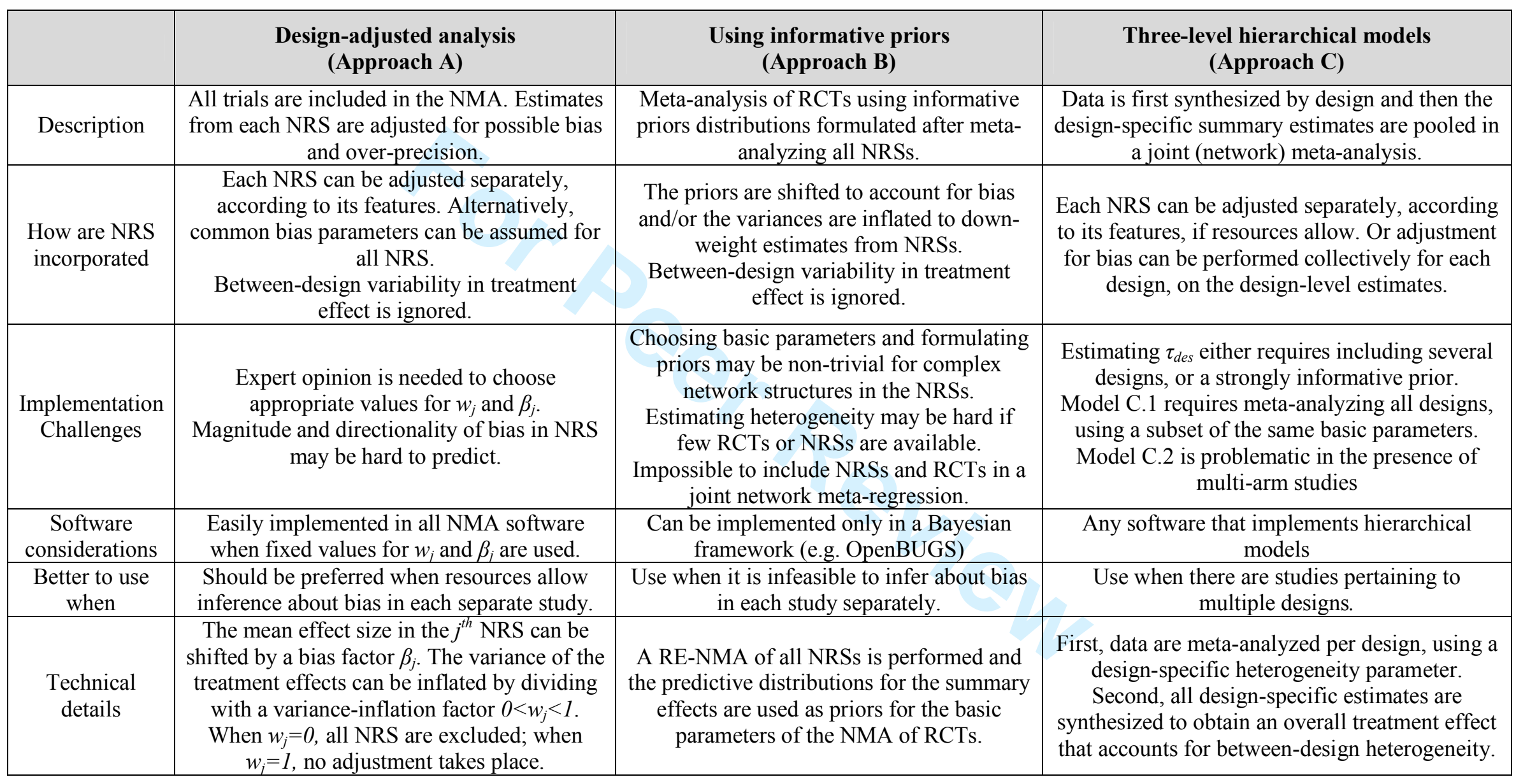


Table 2: Estimated treatment effects and 95\% credible intervals for the antipsychotics network, for basic parameters informed both by RCTs and NRS as estimated from model C1. Larger values of w correspond to larger down-weighting of the NRS.

\begin{tabular}{|c|c|c|c|c|}
\cline { 2 - 5 } \multicolumn{1}{c|}{} & \multicolumn{4}{c|}{ Treatment comparison } \\
\hline Variance inflation factor $(\boldsymbol{w})$ & $\mathbf{1 v 4}$ & $\mathbf{1 v 5}$ & $\mathbf{1 v 6}$ & $\mathbf{1 v 1 5}$ \\
\hline 0.0 & 0.04 & -0.12 & 0.08 & 0.31 \\
(RCTs only) & $(-0.08,0.16)$ & $(-0.26,0.01)$ & $(-0.08,0.24)$ & $(0.11,0.50)$ \\
\hline 0.3 & 0.05 & -0.12 & 0.05 & 0.26 \\
& $(-0.04,0.16)$ & $(-0.23,-0.01)$ & $(-0.08,0.18)$ & $(0.10,0.41)$ \\
\hline 0.7 & 0.06 & -0.11 & 0.04 & 0.25 \\
& $(-0.04,0.16)$ & $(-0.22,-0.01)$ & $(-0.08,0.17)$ & $(0.10,0.40)$ \\
\hline 1.0 & 0.06 & -0.11 & 0.04 & 0.25 \\
(no down-weighting) & $(-0.04,0.16)$ & $(-0.22,0.00)$ & $(-0.08,0.17)$ & $(0.11,0.41)$ \\
\hline
\end{tabular}

Figure 1: Networks of evidence for A) percutaneous interventions for coronary in-stent restenosis, and B) antipsychotics for schizophrenia. Dark grey nodes correspond to treatments compared in RCTs only; light grey nodes are examined in RCTs and NRSs. The size of each node is proportional to the number of studies that examine the corresponding treatment. The thickness of edges is proportional to the number of patients included in the studies that made the corresponding comparison. Codes for percutaneous interventions (Panel A): $A=$ balloon angioplasty, $B=$ bare metal stents, $C=d r u g$-coated balloons, $D=$ everolimus-eluting stents, E=paclitaxel-eluting stents, $F=$ rotablation, $G=$ sirolimus-eluting stents, $H=$ vascular brachytherapy

Figure 2: Relative treatment effects (log OR and their 95\% Confidence Intervals) for target-lesion revascularization with percutaneous interventions for coronary in-stent restenosis, estimated from the various sources of evidence. The results from NMA using only RCTs and from NMA using both RCTs and NRS (naïve NMA) are also presented. Only treatment comparisons informed by both randomized and non-randomized evidence are presented. Codes of interventions as per Figure 1.

Figure 3: Relative treatment effects (Standardized Mean Differences SMD and their 95\% Credible Intervals) for improvement in symptoms scale with antipsychotics in patients with schizophrenia estimated from the various sources of evidence. The results from NMA using only RCTs and from NMA using both RCTs and NRS (naïve NMA) are also presented. Only treatment comparisons informed by both randomized and non-randomized evidence are presented. 
Figure 4: Relative treatment effects (log OR and their 95\% Confidence Intervals) for target-lesion revascularization with percutaneous interventions for coronary in-stent restenosis estimated using approach A. Evidence from the NRSs is given increasing weight with the parameter w. Only treatment comparisons informed by both randomized and non-randomized evidence are presented. Codes of interventions as per Figure 1

Figure 5: Relative treatment effects (Standardized Mean Differences SMD and their 95\% Credible Intervals) for improvement in symptoms scale with antipsychotics in patients with schizophrenia. A large NRS is contributing data to form an informative prior (approach B). Evidence from the NRS is given increasing weight with the parameter $w$. Only treatment comparisons informed by both randomized and non-randomized evidence are presented. 


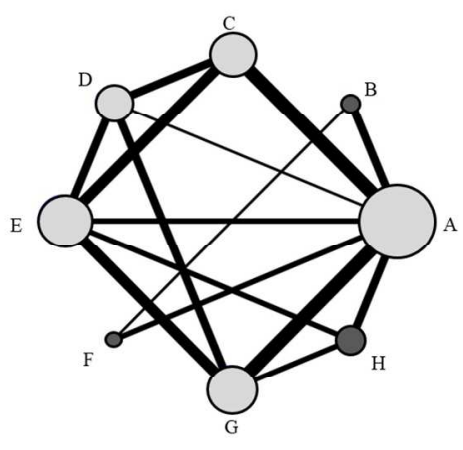

Panel A

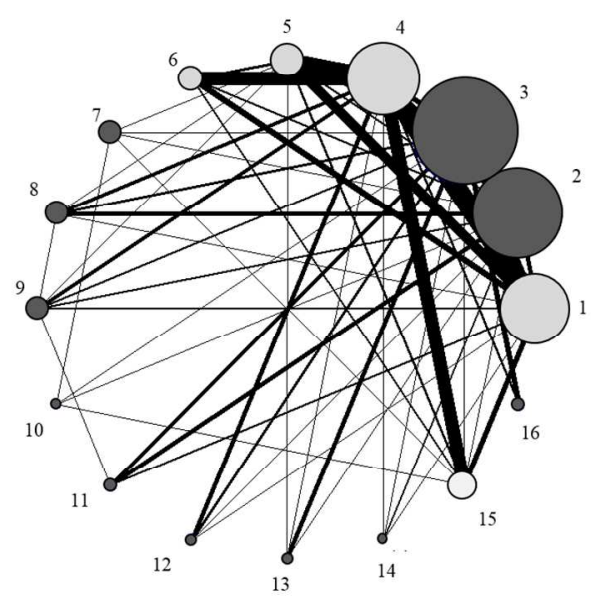

Panel B

Networks of evidence for $A$ ) percutaneous interventions for coronary in-stent restenosis, and B) antipsychotics for schizophrenia. Dark grey nodes correspond to treatments compared in RCTs only; light grey nodes are examined in RCTs and NRSs. The size of each node is proportional to the number of studies that examine the corresponding treatment. The thickness of edges is proportional to the number of patients included in the studies that made the corresponding comparison. Codes for percutaneous interventions (Panel $A$ ): $A=$ balloon angioplasty, $B=$ bare metal stents, $C=$ drug-coated balloons, $D=$ everolimus-eluting stents, $\mathrm{E}=$ paclitaxel-eluting stents, $\mathrm{F}=$ rotablation, $\mathrm{G}=$ sirolimus-eluting stents, $\mathrm{H}=$ vascular brachytherapy Figure 1 


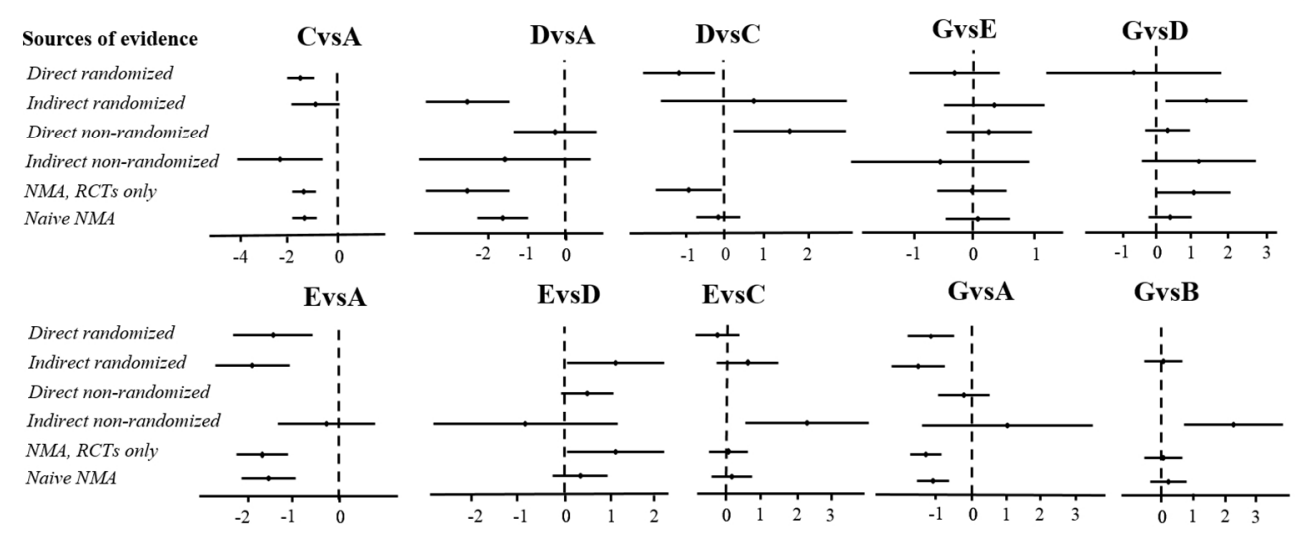

Relative treatment effects (log OR and their 95\% Confidence Intervals) for target-lesion revascularization with percutaneous interventions for coronary in-stent restenosis, estimated from the various sources of evidence. The results from NMA using only RCTs and from NMA using both RCTs and NRS (naïve NMA) are also presented. Only treatment comparisons informed by both randomized and non-randomized evidence are presented. Codes of interventions as per Figure 1.

Figure 2

$130 \times 55 \mathrm{~mm}(300 \times 300 \mathrm{DPI})$ 

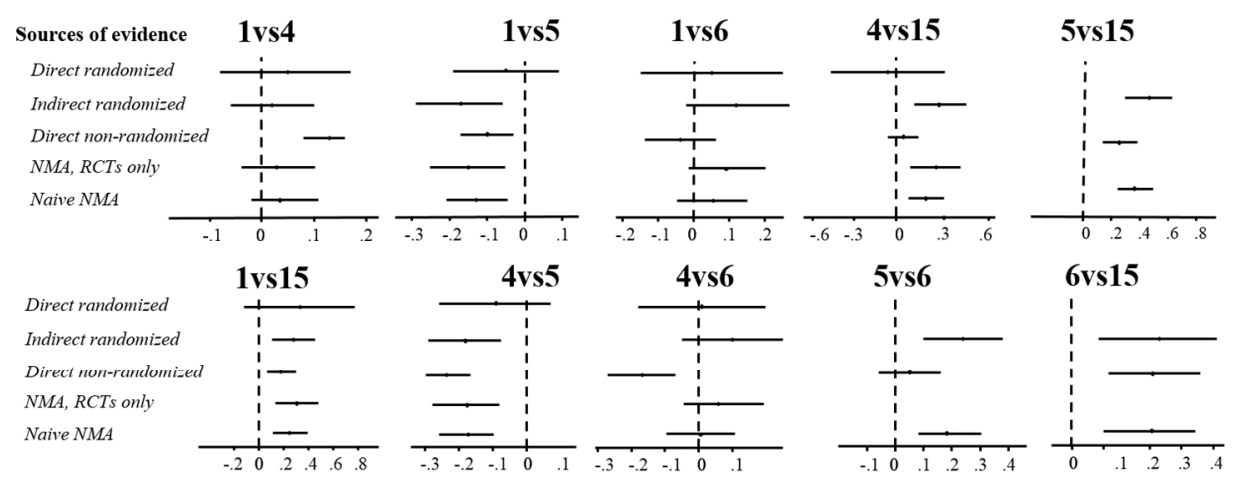

Relative treatment effects (Standardized Mean Differences SMD and their 95\% Credible Intervals) for improvement in symptoms scale with antipsychotics in patients with schizophrenia estimated from the various sources of evidence. The results from NMA using only RCTs and from NMA using both RCTs and NRS

(naïve NMA) are also presented. Only treatment comparisons informed by both randomized and nonrandomized evidence are presented.

Figure 3

$133 \times 52 \mathrm{~mm}(300 \times 300 \mathrm{DPI})$ 
Relative treatment effects (log OR and their 95\% Confidence Intervals) for target-lesion revascularization with percutaneous interventions for coronary in-stent restenosis estimated using approach A. Evidence from the NRSs is given increasing weight with the parameter $w$. Only treatment comparisons informed by both randomized and non-randomized evidence are presented. Codes of interventions as per Figure 1 Figure 4 $135 \times 104 \mathrm{~mm}(300 \times 300$ DPI $)$ 
Relative treatment effects (Standardized Mean Differences SMD and their 95\% Credible Intervals) for improvement in symptoms scale with antipsychotics in patients with schizophrenia. A large NRS is contributing data to form an informative prior (approach B). Evidence from the NRS is given increasing weight with the parameter $\mathrm{w}$. Only treatment comparisons informed by both randomized and nonrandomized evidence are presented.

Figure 5 


\title{
Supplementary material for "Combining randomized and non-randomized evidence in network meta-analysis"
}

\author{
Orestis Efthimiou ${ }^{1}$, Dimitris Mavridis ${ }^{1,2}$, Thomas P. A. Debray ${ }^{3,4}$, M. Samara ${ }^{5}, \mathrm{~S}$ \\ Leucht $^{5}$, M. Belger ${ }^{6}$, George C.M. Siontis ${ }^{7}$ and Georgia Salanti ${ }^{1,8,9}$ on behalf of \\ GetReal Work Package 4
}

\section{Network meta-regression model}

Cooper et al. [1] and Salanti et al. [2] presented a general network meta-regression framework for including study-level covariates in a NMA of aggregated data. Equation (1) of the main paper is now updated as follows:

$$
\begin{gathered}
d_{j X Y} \sim N\left(\theta_{j X Y}, s_{j X Y}^{2}\right) \\
\theta_{j X Y} \sim N\left(\mu_{A Y}-\mu_{A X}+\bar{c}_{j} \beta_{X Y}^{c}, \tau^{2}\right)
\end{gathered}
$$

with $\overline{c_{j}}$ being a study-level covariate and $\beta_{X Y}^{c}$ the corresponding coefficient for the treatment comparison XvsY. Several alternative modeling choices can be made regarding the $\beta$ coefficients. The simplest one is to pick a reference treatment for the regression, e.g. placebo, and to set the coefficients of all treatments versus this reference to be equal. This translates into assuming $\beta_{X P}^{c}=\beta^{c}$ for all treatments $\mathrm{X} \neq \mathrm{P}$, with $\mathrm{P}$ being the reference treatment for the regression. From the consistency equations it then follows that all other $\beta$ parameters are equal to zero. By using this formulation we assume that only relative treatment effects vs. P are influenced by covariate $c$; consequently, the choice of $\mathrm{P}$ becomes important. Note that $\mathrm{P}$ may or may not be the same as the treatment set to be the reference when picking the basic parameters of the model (this treatment can be arbitrarily chosen). Details about

\footnotetext{
${ }^{1}$ Department of Hygiene and Epidemiology, University of Ioannina School of Medicine, Ioannina, Greece

${ }^{2}$ Department of Primary Education, University of Ioannina, Ioannina, Greece

${ }^{3}$ Julius Center for Health Sciences and Primary Care, University Medical Center Utrecht, Utrecht, The Netherlands

${ }^{4}$ Cochrane Netherlands, Julius Center for Health Sciences and Primary Care, University Medical Center Utrecht, Utrecht, The Netherlands

${ }^{5}$ Department of Psychiatry and Psychotherapy, Technische Universität München, München, Germany

${ }^{6}$ Eli Lilly and Company, Lilly Research Centre, Windlesham, UK

${ }^{7}$ Department of Cardiology, Bern University Hospital, Bern, Switzerland

${ }^{8}$ Institute of Social and Preventive Medicine, University of Bern, Bern, Switzerland

${ }^{9}$ Berner Institut für Hausarztmedizin (BIHAM), University of Bern, Bern, Switzerland
} 
alternative modeling choices can be in the original publications [1, 2] and also in our recent review [3].

An issue that may come up when using network meta-regression in practice is the issue of missing covariate data: in our case study, for example, the mean age and the mean duration of illness of participants are deemed to be important study-level effect modifiers. There are studies reporting mean age and duration, only age, only duration or none of these two covariates. Analyzing only the complete cases (studies reporting both covariates) would exclude from the analysis a significant number of studies. We can include in the network meta-regression model studies with missing covariates after using multiple imputations for the missing values. A simple way to stochastically impute values in a Bayesian setting is to draw values from a distribution centered on the mean of the available data. For example we can stochastically impute a missing value for the mean participant age in study $j$ by assuming $\overline{a g e}_{j} \sim N\left(\hat{\mu}_{\overline{a g e}}, \hat{s}_{\overline{a g e}}^{2}\right)$, where $\hat{\mu}_{\overline{a g e}}$ and $\hat{s}_{\overline{a g e}}$ are the mean value and standard deviation of the mean age in studies that report it.

This imputation method overlooks the fact that some covariates may be dependent on others. In our case study, for example, mean participant age and duration of illness are highly correlated. To also take this into account we can assume a model that draws the missing covariate values from a multivariate normal distribution centered on the mean of the available cases. More specifically we can assume that the mean age $\left(\overline{a g e}_{j}\right)$ and mean duration of illness $\left(\overline{d u r}_{j}\right)$ follow from:

$$
\left(\begin{array}{c}
\overline{a g e}_{j} \\
\overline{d u r}_{j}
\end{array}\right) \sim N\left(\left(\begin{array}{cc}
\hat{\mu}_{\overline{a g e}} \\
\hat{\mu} \overline{d u r}
\end{array}\right),\left(\begin{array}{cc}
\hat{s}_{\overline{a g e}}^{2} & \rho_{a g e-d u r} \hat{s}_{\overline{a g e}} \hat{s}_{\overline{d u r}} \\
\rho_{\text {age-dur }} \hat{s}_{\overline{a g e}} \hat{s}_{\overline{d u r}} & \hat{s}_{\overline{d u r}}^{2}
\end{array}\right)\right)
$$

The full network meta-regression model for a 2-arm study $j$ is the following:

$$
\begin{gathered}
d_{j X Y} \sim N\left(\theta_{j X Y}, s_{j}^{2}\right) \\
\theta_{j X Y} \sim N\left(\mu_{A Y}-\mu_{A X}+\overline{a g e}_{j} \beta_{X Y}^{a g e}+\overline{d u r}_{j} \beta_{X Y}^{d u r}, \tau^{2}\right)
\end{gathered}
$$

where $\beta_{T P}^{a g e}=\beta^{a g e}$ and $\beta_{T P}^{\text {dur }}=\beta^{\text {dur }} \forall T \neq P$, while $\beta_{T T}^{a g e}=\beta_{T T}^{\text {dur }}=0 \forall T$. 


\section{Issues with Approach B (using non-randomized evidence as prior information)}

In this approach we perform an NMA in the RCTs, with the model being as in Equation (1) of the main paper, but we now assume informative prior distributions for the basic parameters. These follow from the analysis of the non-randomized data, after possibly shifting the mean and/or inflating the variance, e.g. $\mu_{A X} \sim N\left(\hat{\mu}_{A X}^{N R S}+\right.$ $\left.\beta_{A X}, \widehat{V}_{A X}^{N R S} / w_{A X}\right)$.

If the non-randomized evidence forms a network of evidence, however, formulating prior distributions for the basic parameters of the NMA of RCTs may become a non-trivial matter. To clarify this issue let us consider the example depicted in Figure 1 below. This shows a network of randomized studies comparing treatments A-H. Seven basic parameters need to be included in the model (equal to the number of treatments minus one). Let us assume that there are some NRSs informing $\mathrm{AB}$, some NRSs informing CD, and a network of NRSs informing the comparisons between treatments E, F, G and H (thick black lines in Figure 1). In the first step we analyze all non-randomized evidence, i.e. we perform meta-analyses of the $\mathrm{AB}$ and $\mathrm{CD}$ studies and a NMA of the studies comparing E, F, G and H. For considerations regarding how to model heterogeneity in such cases we refer the reader to the main paper.

In the second step, we use the estimates obtained from the first step to formulate predictive prior distributions for the basic parameters of the NMA of the RCTs. If for example treatment $\mathrm{A}$ is chosen to be the reference, then for the basic parameters AC and $\mathrm{AD}$ the prior distribution should be a bivariate normal distribution with parameters carefully chosen so that they carry no information about $\mathrm{AC}$ and $\mathrm{AD}$, but so that they do carry information about $\mathrm{CD}$ :

$$
\left(\begin{array}{l}
\mu_{A C} \\
\mu_{A D}
\end{array}\right) \sim N\left(\left(\begin{array}{c}
d \\
d+\hat{\mu}_{C D}^{N R S}
\end{array}\right),\left(\begin{array}{cc}
V_{1} & \operatorname{Cov} \\
\operatorname{Cov} & V_{2}
\end{array}\right)\right)
$$

where $d$ is an arbitrary constant, e.g. it can be set equal to zero with no loss of generality. Given that $\mu_{C D}=\mu_{A D}-\mu_{A C}$, the configuration above ensures that the prior information about $\mathrm{CD}$ is centered on $\hat{\mu}_{C D}^{N R S}$, exactly as it should. Constants $V_{1}$ and $V_{2}$ should be large enough (e.g. $V_{1}=V_{2}=1000^{2}$ ) to ensure that the marginal distributions for $\mu_{A C}$ and $\mu_{A D}$ remain uninformative (since the non-randomized evidence provides no information about $\mathrm{AC}$ or $\mathrm{AD})$. Given that $\operatorname{Var}\left(\mu_{C D}\right)=$ $\operatorname{Var}\left(\mu_{A D}\right)+\operatorname{Var}\left(\mu_{A C}\right)-2 \operatorname{Cov}\left(\mu_{A D}, \mu_{A C}\right)$, it follows that the $\operatorname{Cov}$ parameter of 
Equation (2) should be set equal to $\operatorname{Cov}=\left(V_{1}+V_{2}-\widehat{V}_{C D}^{N R S}\right) / 2$. This choice ensures that Equation (2) incorporates the correct amount of uncertainty for the $\mathrm{CD}$ comparison. Similarly, for the case of the basic parameters AE, AF, AG and AH a multivariate normal distribution with 4 components needs to be used, with the variance-covariance matrix carefully structured so as to include the information conveyed in the non-randomized studies regarding EF, EG, EH, FG, FH and GH.

One can greatly simplify this complicated process by cleverly choosing the basic parameters. This is depicted using red lines in Figure 1. The non-randomized evidence can provide information about five basic parameters, so we choose $\mathrm{AB}, \mathrm{CD}, \mathrm{EF}, \mathrm{EG}$, EH. For $\mathrm{AB}$ and $\mathrm{CD}$ we need univariate normal distributions, i.e. $\left(\hat{\mu}_{A B}^{N R S}, \widehat{V}_{A B}^{N R S}\right)$ and $N\left(\hat{\mu}_{C D}^{N R S}, \hat{V}_{C D}^{N R S}\right)$ respectively. EF, EG and EH will be correlated so that multivariate normal distributions need to be used, as estimated from the non-randomized data, without any further complications. Two more basic parameters are needed, e.g. we can choose $\mathrm{BC}$ and $\mathrm{DE}$, to which vague prior distributions need to be assigned.

Figure 1: A network of eight treatments (A-H) including both randomized and nonrandomized studies. Thin black lines correspond to treatment comparisons for which only randomized evidence is available. Thick black lines correspond to comparisons for which non-randomized evidence is also available. Dashed red lines correspond to a possible choice of the seven basic parameters needed for the NMA model that uses non-randomized evidence as prior information. The basic parameters form a 'spanning tree': they pass through all treatments and do not form any loops. Other choices of basic parameters are valid as well, e.g. AH could replace DE.

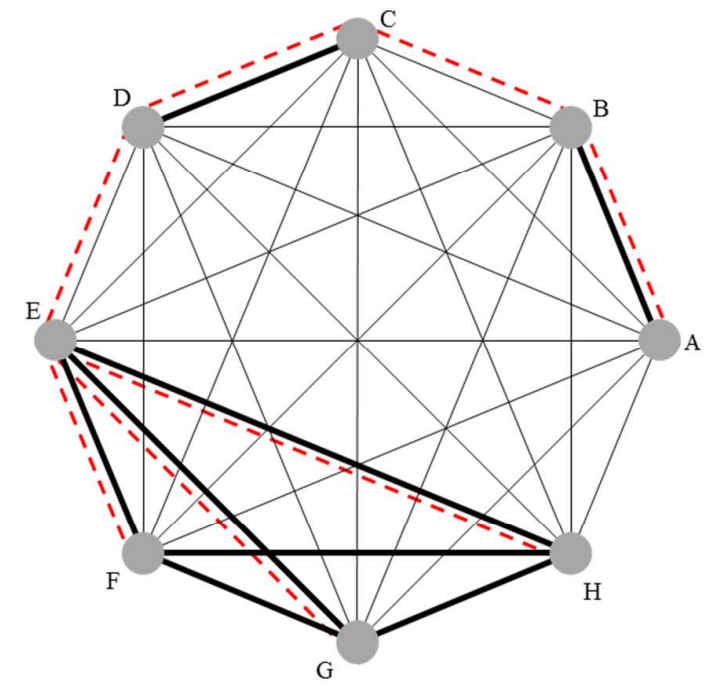




\section{Relation between the mixture parameter and the variance inflation factor}

In Section 3.3 of the main paper we discuss three approaches for using nonrandomized evidence as prior information. Here we discuss how the mixture parameter of the third approach relates to the variance inflation factor $w$, for the special case of normal distributions.

Let us focus to the case of a mixture prior for a single parameter. Assume that the informative part of the mixture prior (based on the non-randomized evidence) is $N\left(\mu_{N R S}, V_{N R S}\right)$, and let us also assume that the uninformative part is $N\left(0, V_{0}\right)$. The mixture prior is a linear combination of these two normal distributions, where parameter $p$ controls the mixing. For fixed $p$ this mixture prior is also a normal distribution, with variance equal to $p^{2} V_{N R S}+(1-p)^{2} V_{0}$.

If instead of a mixture we use a variance inflation factor (w), the variance of the prior distribution is $V_{N R S} / w$. By setting $p^{2} V_{N R S}+(1-p)^{2} V_{0}=V_{N R S} / w$ we can calculate how the mixture parameter relates to $\mathrm{w}$, i.e. what values of $w$ and $p$ lead to the same prior distribution. By solving for $w$ we get $w=N_{N R S}\left(p^{2} V_{N R S}+\right.$ $\left.(1-p)^{2} V_{0}\right)^{-1}$.

\section{Approach C (three-level hierarchical models)}

Here we discuss the three-level hierarchical models presented in the main paper. For all three models we assume that at the first level the study $j$ of the $i^{\text {th }}$ design, compares $\mathrm{X}$ vs. $\mathrm{Y}$ and it is analyzed separately to provide an estimate of the relative effect $d_{i j X Y}$, with a standard error $s_{i j X Y}$.

\subsection{Model C.1}

For model C.1 at the second level a NMA is performed for each design, i.e. we assume

$$
\begin{gathered}
d_{i j X Y} \sim N\left(\theta_{i j X Y}, s_{i j X Y}^{2}\right) \\
\theta_{i j X Y} \sim N\left(\kappa_{i A Y}-\kappa_{i A X}, \tau_{i}^{2}\right)
\end{gathered}
$$

where $\kappa_{i A X}, \kappa_{i A Y}, \ldots$ are the basic parameters and $\tau_{i}^{2}$ is the heterogeneity variance of the $i^{\text {th }}$ design. The model can be extended to include multi-arm studies using standard NMA methodology. At the third level the basic parameters are assumed to be exchangeable across designs, i.e.

$$
\kappa_{i A X} \sim N\left(\mu_{A X}, \tau_{d e s}^{2}\right)
$$




$$
\kappa_{i A Y} \sim N\left(\mu_{A Y}, \tau_{d e s}^{2}\right)
$$

All other relative treatment effects can be calculated by combining the estimates on the various $\kappa$, e.g. $\mu_{X Y}=\mu_{A Y}-\mu_{A X}$.

Note that for the NMA of each design we use as basic parameters a subset of the basic parameters synthesized at the third level. e.g. a design may provide information on basic parameters $\mathrm{AB}$ and $\mathrm{AC}$, another design information on $\mathrm{AB}$ and $\mathrm{AD}$ etc.. This model will not be applicable in some scenarios. Let us consider the example depicted in Figure 2, where there are four treatments in total (A, B, C and D) and there are studies pertaining to three different designs. For the NMA of the first design one could use as basic parameters any two of the following three: $\mathrm{AB}, \mathrm{AC}$ or $\mathrm{BC}$. Similarly, for the second design any two of $\mathrm{AB}, \mathrm{AD}$ and $\mathrm{BD}$, while for the third design any two comparisons out of $\mathrm{BC}, \mathrm{BD}$ and $\mathrm{CD}$. It is evident that one cannot find a set of three basic parameters (number of total treatments minus one) so that every design could be meta-analyzed using a subset of this set.

Figure 2: A scenario for which model C.1 could not be used.

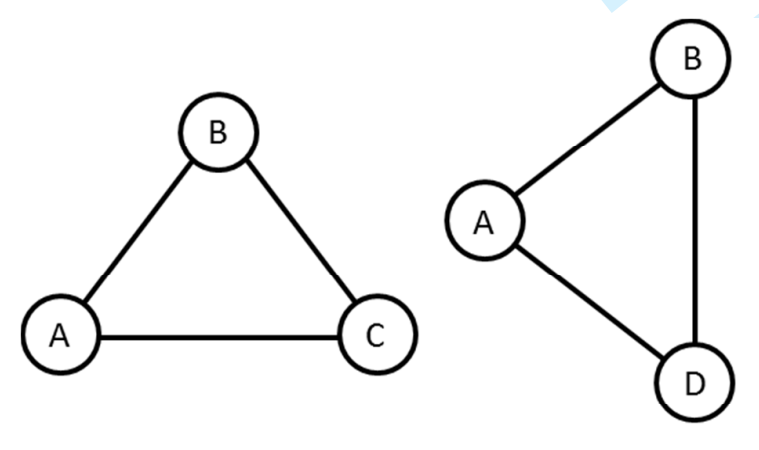

design 1

design 2

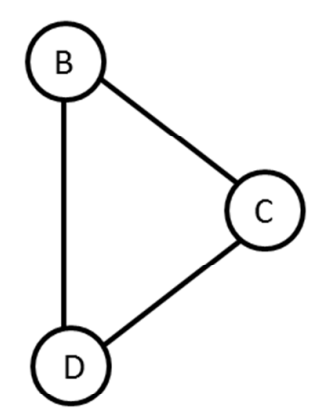

design 3

\subsection{Model C.2}

For model C.2 at the second level a simple, pairwise meta-analysis is performed for each comparison in each design, sharing however the same heterogeneity variance, i.e. we assume

$$
\begin{gathered}
d_{i j X Y} \sim N\left(\theta_{i j X Y}, s_{i j X Y}^{2}\right) \\
\theta_{i j X Y} \sim N\left(\kappa_{i X Y}, \tau_{i}^{2}\right)
\end{gathered}
$$


This is in essence the same model as in Equation (3), after excluding the consistency equations; this has been termed as the unrelated mean effects (UME) model [4]. If comparison-specific heterogeneities are assumed, this equation describes a series of unrelated pairwise meta-analyses.

At the third level we synthesize all design-specific estimates of the second level into an NMA, allowing for design-level heterogeneity, i.e. we assume that:

$$
\kappa_{i X Y} \sim N\left(\mu_{A Y}-\mu_{A X}, \tau_{d e s}^{2}\right)
$$

This model can handle scenarios like the one presented in Figure 2. In this example at the third level we would perform a NMA on the estimates obtained at the second level: $\kappa_{1 A B}, \kappa_{1 A C}, \kappa_{1 B C}$ (from the $1^{\text {st }}$ design), $\kappa_{2 A B}, \kappa_{2 A D}, \kappa_{2 B D}$ (from the $2^{\text {nd }}$ design) and $\kappa_{3 B C}, \kappa_{3 B D}, \kappa_{3 C D}$ (from the $3^{\text {rd }}$ design). These nine estimates would be analyzed at the third level as if they were obtained from a single study each.

When multi-arm studies are present model C.2, becomes problematic.

\subsection{Model C.3}

For model C.3 at the second level a NMA is performed per design, imposing the consistency equations, exactly as in Equation (3). The output from each NMA is a vector of the estimated treatment effects, with a corresponding variance-covariance matrix. These estimates are used for the NMA at the third level, treated as if they belonged to multi-arm studies. For the example of Figure 2 at the second level we would estimate $\boldsymbol{\kappa}_{\mathbf{1}}=\left(\kappa_{1 A B}, \kappa_{1 A C}\right)^{\prime}$ from design $1, \boldsymbol{\kappa}_{\mathbf{2}}=\left(\kappa_{2 A B}, \kappa_{2 B D}\right)^{\prime}$ from design 2 and $\boldsymbol{\kappa}_{\mathbf{3}}=\left(\kappa_{3 B D}, \kappa_{3 C D}\right)^{\prime}$ from design 3 , along with $\boldsymbol{C}_{\mathbf{1}}, \boldsymbol{C}_{\mathbf{2}}$ and $\boldsymbol{C}_{\mathbf{3}}$, the three variancecovariance matrices. Note that the choice of basic parameters in the NMA of each design is arbitrary, different choices will not change results. At the third level a NMA is again performed using these three estimates as if they belonged to a single multiarm trial each. For the running example we would assume that

$$
\left(\begin{array}{l}
\kappa_{1 A B} \\
\kappa_{1 A C} \\
\kappa_{2 A B} \\
\kappa_{2 B D} \\
\kappa_{3 B D} \\
\kappa_{3 C D}
\end{array}\right) \sim N\left(\left(\begin{array}{l}
\lambda_{1 A B} \\
\lambda_{1 A C} \\
\lambda_{2 A B} \\
\lambda_{2 B D} \\
\lambda_{3 B D} \\
\lambda_{3 C D}
\end{array}\right), \operatorname{Diag}\left(\boldsymbol{C}_{\mathbf{1}} \boldsymbol{C}_{2}, \boldsymbol{C}_{\mathbf{3}}\right)\right.
$$

and 
1

2

3

4

5

6

7

8

9

10

11

12

13

14

15

16

17

18

19

20

21

22

23

24

25

26

27

28

29

30

31

32

33

34

35

36

37

38

39

40

41

42

43

44

45

46

47

48

49

50

51

52

53

54

55

56

57

58

59

60

$$
\left(\begin{array}{l}
\lambda_{1 A B} \\
\lambda_{1 A C} \\
\lambda_{2 A B} \\
\lambda_{2 B D} \\
\lambda_{3 B D} \\
\lambda_{3 C D}
\end{array}\right) \sim N\left(\left(\begin{array}{c}
\mu_{A B} \\
\mu_{A C} \\
\mu_{A B} \\
\mu_{A D}-\mu_{A B} \\
\mu_{A D}-\mu_{A B} \\
\mu_{A D}-\mu_{A C}
\end{array}\right), \operatorname{Diag}(\boldsymbol{T}, \boldsymbol{T}, \boldsymbol{T})\right.
$$

where $\boldsymbol{T}=\left(\begin{array}{cc}\tau_{\text {des }}^{2} & \tau_{\text {des }}^{2} / 2 \\ \tau_{\text {des }}^{2} / 2 & \tau_{\text {des }}^{2}\end{array}\right)$ is the design-level heterogeneity variance-covariance matrix. Note that the consistency equations are used at both the second and the third level.

In all three models estimates from the NRSs can be adjusted either at the study level (by shifting the mean and/or inflating the variance) or at the design level, i.e. by adjusting the output of the design-level estimates separately for each design. In Figure 3 we provide a schematic representation of the three alternative three-level hierarchical models presented in this paper. 
Figure 3: Schematic representation of the three-level hierarchical models. Abbreviations: $M A=$ meta-analysis, $N M A=$ network meta-analysis

Model C.1: an NMA per design at the $2^{\text {nd }}$ level, an MA per basic parameter at the $3^{\text {rd }}$ level

Model C.2: a MA per design at the $2^{\text {nd }}$ level, an overall NMA at the $3^{\text {rd }}$ level Model C.3: an NMA per design at the $2^{\text {nd }}$ level, an overall NMA at the $3^{\text {rd }}$ level.

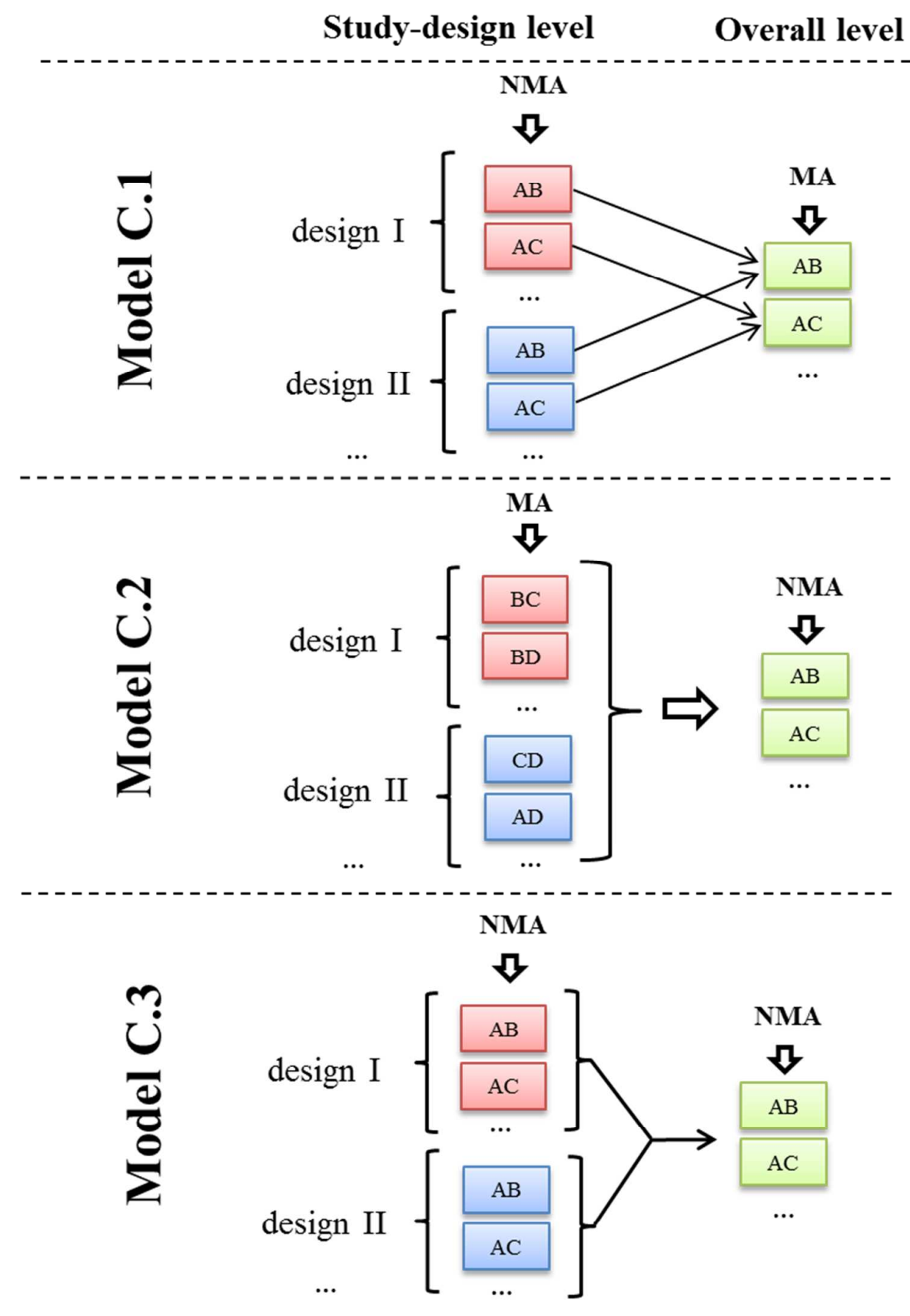




\section{Percutaneous interventions for the treatment of coronary in-stent restenosis example}

In this section we present some results from the analysis of the coronary in-stent restenosis network. The NRSs that we included in this analysis are given in the reference list of this appendix [5-10].

In order to assess the inconsistency we applied the loop-specific and the nodesplitting approaches. In Table 1 we present the results of the loop-specific approach for identifying inconsistency, for the case of the NMA with RCTs only. In Table 2 we give results for the naïve NMA. The analysis were performed using the ifplot command in Stata. The inconsistency factors (IF) presented in these tables correspond to the difference between direct and indirect evidence in each loop.

Table 1: Inconsistency factors - NMA with RCTs only

\begin{tabular}{|c|c|c|c|c|c|c|c|c|}
\hline 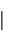 & Loop & IF & seIF & I z_value & I p_value & CI_9 & & tau2 \\
\hline & & & ---- & & & & & \\
\hline & $A-C-D-G$ & | 2.312 & 1.508 & 1.533 & 0.125 & $(0.00,5.27)$ & I & 0.189 \\
\hline & $C-D-E-G$ & 1.537 & 1.679 & 0.915 & 0.360 & $(0.00,4.83)$ & 1 & 0.369 \\
\hline & $A-C-E$ & 0.939 & 0.739 & 1.271 & 0.204 & $(0.00,2.39)$ & 1 & 0.282 \\
\hline & $A-E-G$ & 0.464 & 0.729 & 0.637 & 0.524 & $(0.00,1.89)$ & | & 0.218 \\
\hline & $\mathrm{E}-\mathrm{G}-\mathrm{H}$ & 10.337 & 1.038 & 0.324 & 0.746 & $(0.00,2.37)$ & 1 & 0.439 \\
\hline & $A-E-H$ & | 0.277 & | 0.445 & 0.623 & 0.533 & $\mid(0.00,1.15)$ & 1 & 0.019 \\
\hline & $A-B-F$ & 0.138 & | 0.992 & 0.139 & 0.889 & $1(0.00,2.08)$ & | & 0.281 \\
\hline & $A-G-H$ & 10.092 & 0.416 & 0.220 & 0.826 & $(0.00,0.91)$ & 1 & 0.022 \\
\hline
\end{tabular}

Table 2: Inconsistency factors - naïve NMA

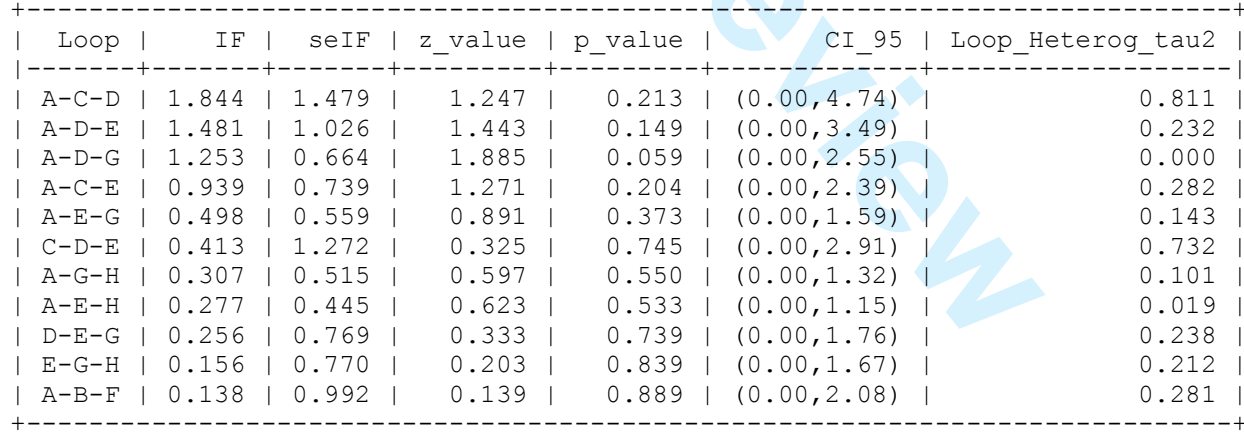

In Table 3 we give results from the node-splitting approach for assessing inconsistency, before and after the inclusion of NRSs in the network. Analyses were performed using the network sidesplit command in Stata. 
Table 3: Estimates from the node-splitting approach for assessing inconsistency.

\begin{tabular}{|c|c|c|}
\cline { 2 - 3 } \multicolumn{1}{c|}{} & \multicolumn{2}{c|}{ difference between direct and indirect evidence } \\
(standard error)
\end{tabular}

In Table 4 we give the estimates for the heterogeneity variance $\left(\tau^{2}\right)$ for the analyses discussed in Section 5.2 of the main paper.

Table 4: Estimates for the heterogeneity variance (assumed common in the network)

\begin{tabular}{|c|c|}
\hline Analysis (approach A) & Heterogeneity $\left(\boldsymbol{\tau}^{2}\right)$ \\
\hline RCTs only & 0.13 \\
\hline$w=0.2$ & 0.12 \\
\hline$w=0.5$ & 0.14 \\
\hline$w=0.8$ & 0.18 \\
\hline Naïve analysis $(w=1)$ & 0.20 \\
\hline
\end{tabular}




\section{Using model $\mathrm{C}$ for the antipsychotics example}

As we discussed in Section 3.3 of the main paper, approach $\mathrm{C}$ requires the availability of multiple study designs. We were, however, unable to identify any suitable example to use for illustrating the method. Thus, in order to exemplify approach $\mathrm{C}$ we used the antipsychotics example after splitting the available RCTs in groups and treating each of these groups as if it pertained to a different design. We do the splitting according to the risk of bias (RoB) assessment that was performed in the original publication [11]. The authors had assessed each RCT as being of "high RoB", "low RoB" or "unclear", for each one of the following five bias domains: randomization, allocation, blinding, missing outcomes and other biases. Using this assessment, we created three groups. The first group ('high RoB RCTs') incorporated all studies judged to be at a high RoB for at least one of the five bias domains; this group included 97 RCTs. The second group ('low RoB RCTs') included studies judged to be at a low RoB for at least four of the five bias domains; only 18 studies were included in this group. The rest of the studies were grouped into a 'unclear RoB' group of RCTs; there were 52 such studies. We then treated the groups as being different designs, and we assumed the single NRS to be of a different design. We used model $\mathrm{C} 1$ presented in Section 3.3 to synthesize the data.

\section{Results for the antipsychotics example}

\subsection{Results from the naïve analysis}

In Table 5 we give the estimates from all model parameters of the naïve NMA. The regression coefficients correspond to the comparison of all active drugs vs. placebo. In Tables 2 and 3 we give the results from the inconsistency factors (i.e. the difference between direct and indirect evidence in each loop) for all loops in the network, before and after the inclusion of the observational study. In Table 8 we present results from the node-splitting approach for assessing inconsistency.

Table 5: Naïve analysis - estimates of the model parameters

\begin{tabular}{|c|c|}
\hline Parameter & Median estimate (95\% Cr.I.) \\
\hline$\tau$ & 0.085 \\
(heterogeneity SD) & $(0.052,0.120)$ \\
\hline$\beta^{\text {age }}$ & 0.10 \\
(regression coefficient for mean age) & $(-0.10,0.28)$ \\
\hline$\beta^{\text {dur }}$ & 0.00 \\
(regression coefficient for mean duration of illness) & $(-0.22,0.22)$ \\
\hline
\end{tabular}




\begin{tabular}{|c|c|}
\hline $\begin{array}{c}\rho_{a g e-d u r} \\
\text { (correlation coefficient between mean age and mean } \\
\text { duration of illness) }\end{array}$ & $\begin{array}{c}0.89 \\
(0.85,0.92)\end{array}$ \\
\hline
\end{tabular}

Table 6: Inconsistency factors - NMA with RCT only

\begin{tabular}{|c|c|c|c|c|c|c|c|c|c|c|c|c|}
\hline & Loop & । & $\mathrm{IF}$ & | & seIF & | & lue & & ralue & CI_95 & \\
\hline 1 & & 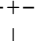 & & & & & & 1 & & 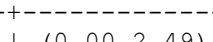 & & \\
\hline | & $3-4-15$ & 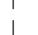 & $\begin{array}{l}1.235 \\
1.119\end{array}$ & 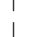 & 0.639 & 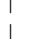 & $\begin{array}{l}1.933 \\
1.825\end{array}$ & 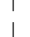 & 0.053 & $\begin{array}{r}(0.00,2.49) \\
(0.00 .2 .32)\end{array}$ & 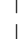 & 0.081 \\
\hline I & $3-7-10$ & i & 0.810 & i & 0.271 & | & 2.986 & i & 0.003 & $(0.28,1.34)$ & i & 0.000 \\
\hline | & $3-7-15$ & | & 0.772 & । & 0.596 & | & 1.296 & । & 0.195 & I $(0.00,1.94)$ & । & 0.059 \\
\hline | & $1-3-15$ & I & 0.734 & । & 0.571 & | & 1.285 & । & 0.199 & I $(0.00,1.85)$ & I & 0.005 \\
\hline | & $3-10-15$ & 1 & 0.732 & 1 & 0.698 & 1 & 1.049 & । & 0.294 & $(0.00,2.10)$ & i & 0.070 \\
\hline & $7-10-15$ & I & 0.707 & 1 & 0.505 & 1 & 1.401 & । & 0.161 & I $(0.00,1.70)$ & I & 0.092 \\
\hline | & $2-3-15$ & | & 0.677 & 1 & 0.553 & | & 1.224 & | & 0.221 & I $(0.00,1.76)$ & | & 0.018 \\
\hline | & $1-3-12$ & 1 & 0.567 & I & 0.258 & 1 & 2.203 & । & 0.028 & I $(0.06,1.07)$ & I & 0.013 \\
\hline | & $5-8-9$ & | & 0.529 & 1 & 0.352 & | & 1.500 & । & 0.134 & I $(0.00,1.22)$ & I & 0.000 \\
\hline | & $2-5-8$ & 1 & 0.504 & 1 & 0.327 & | & 1.538 & । & 0.124 & I $(0.00,1.15)$ & । & 0.000 \\
\hline | & $1-2-14$ & | & 0.451 & 1 & 0.323 & | & 1.397 & । & 0.163 & I $(0.00,1.08)$ & । & 0.036 \\
\hline | & $2-5-7$ & I & 0.443 & 1 & 0.438 & | & 1.010 & । & 0.312 & I $(0.00,1.30)$ & I & 0.000 \\
\hline | & $2-4-15$ & I & 0.415 & । & 0.273 & I & 1.520 & । & 0.128 & $1(0.00,0.95)$ & I & 0.015 \\
\hline 1 & $3-5-8$ & । & 0.413 & 1 & 0.369 & i & 1.119 & । & 0.263 & $(0.00,1.14)$ & I & 0.023 \\
\hline | & $2-3-7$ & । & 0.408 & 1 & 0.438 & | & 0.931 & । & 0.352 & I $(0.00,1.27)$ & । & 0.009 \\
\hline | & $1-3-14$ & | & 0.378 & 1 & 0.201 & | & 1.881 & | & 0.060 & I $(0.00,0.77)$ & | & 0.004 \\
\hline | & $1-4-15$ & I & 0.368 & । & 0.299 & 1 & 1.229 & I & 0.219 & $1(0.00,0.95)$ & I & 0.000 \\
\hline | & $1-5-8$ & 1 & 0.362 & । & 0.336 & 1 & 1.078 & | & 0.281 & I $(0.00,1.02)$ & | & 0.000 \\
\hline | & $2-3-12$ & I & 0.361 & 1 & 0.197 & 1 & 1.835 & । & 0.067 & $1(0.00,0.75)$ & I & 0.013 \\
\hline | & $3-4-12$ & । & 0.341 & 1 & 0.160 & 1 & 2.137 & । & 0.033 & $(0.03,0.65)$ & । & 0.019 \\
\hline | & $1-4-14$ & । & 0.334 & । & 0.191 & | & 1.749 & । & 0.080 & $1(0.00,0.71)$ & । & 0.000 \\
\hline | & $4-5-7-15$ & । & 0.320 & 1 & 0.307 & | & 1.044 & । & 0.297 & $1(0.00,0.92)$ & | & 0.007 \\
\hline 1 & $1-2-12$ & | & 0.306 & । & 0.436 & | & 0.701 & 1 & 0.483 & I $(0.00,1.16)$ & I & 0.048 \\
\hline | & $2-7-15$ & I & 0.286 & । & 0.574 & | & 0.498 & 1 & 0.618 & I $(0.00,1.41)$ & I & 0.095 \\
\hline | & $2-4-13$ & i & 0.286 & 1 & 0.188 & 1 & 1.519 & 1 & 0.129 & $(0.00,0.65)$ & i & 0.000 \\
\hline | & $2-10-15$ & | & 0.251 & I & 0.407 & | & 0.617 & 1 & 0.537 & $\mid(0.00,1.05)$ & 1 & 0.102 \\
\hline | & $4-5-8$ & | & 0.249 & | & 0.326 & | & 0.763 & 1 & 0.446 & I $(0.00,0.89)$ & | & 0.000 \\
\hline | & $4-8-9$ & 1 & 0.246 & । & 0.148 & 1 & 1.661 & । & 0.097 & I $(0.00,0.54)$ & । & 0.000 \\
\hline | & $1-2-9$ & | & 0.217 & 1 & 0.250 & | & 0.870 & । & 0.384 & $(0.00,0.71)$ & i & 0.036 \\
\hline | & $1-4-12$ & | & 0.199 & 1 & 0.202 & | & 0.988 & I & 0.323 & $1(0.00,0.59)$ & । & 0.000 \\
\hline i & $1-9-11$ & i & 0.196 & i & 0.154 & i & 1.276 & i & 0.202 & $1(0.00,0.50)$ & i & 0.001 \\
\hline 1 & $1-2-5$ & I & 0.195 & 1 & 0.177 & I & 1.105 & । & 0.269 & I $(0.00,0.54)$ & I & 0.022 \\
\hline i & $2-4-5$ & i & 0.176 & i & 0.102 & i & 1.719 & i & 0.086 & $(0.00,0.38)$ & i & 0.000 \\
\hline | & $2-3-11$ & 1 & 0.173 & 1 & 0.087 & | & 1.983 & | & 0.047 & $1(0.00,0.34)$ & I & 0.000 \\
\hline | & $1-8-9$ & 1 & 0.171 & 1 & 0.179 & 1 & 0.952 & | & 0.341 & I $(0.00,0.52)$ & | & 0.000 \\
\hline | & $3-5-13$ & I & 0.157 & I & 0.261 & | & 0.602 & । & 0.547 & $(0.00,0.67)$ & I & 0.037 \\
\hline | & $3-9-11$ & | & 0.156 & । & 0.148 & | & 1.055 & । & 0.291 & $(0.00,0.45)$ & | & 0.000 \\
\hline | & $2-4-9$ & 1 & 0.155 & 1 & 0.101 & | & 1.531 & । & 0.126 & $1(0.00,0.35)$ & I & 0.000 \\
\hline | & $2-4-6$ & I & 0.148 & । & 0.154 & 1 & 0.961 & । & 0.336 & $1(0.00,0.45)$ & 1 & 0.009 \\
\hline i & $1-3-5$ & i & 0.117 & i & 0.127 & i & 0.918 & । & 0.358 & $(0.00,0.37)$ & i & 0.017 \\
\hline | & $1-3-9$ & 1 & 0.117 & 1 & 0.122 & 1 & 0.955 & । & 0.339 & $1(0.00,0.36)$ & 1 & 0.003 \\
\hline i & $3-4-13$ & i & 0.109 & i & 0.295 & i & 0.371 & i & 0.710 & $(0.00,0.69)$ & i & 0.054 \\
\hline | & $2-3-10$ & I & 0.106 & 1 & 0.218 & | & 0.484 & । & 0.628 & $1(0.00,0.53)$ & । & 0.012 \\
\hline i & $3-8-9$ & 1 & 0.103 & i & 0.158 & i & 0.649 & i & 0.516 & $(0.00,0.41)$ & i & 0.000 \\
\hline | & $1-2-3$ & 1 & 0.098 & 1 & 0.113 & 1 & 0.867 & | & 0.386 & $1(0.00,0.32)$ & | & 0.020 \\
\hline | & $3-4-8$ & I & 0.097 & 1 & 0.147 & | & 0.658 & | & 0.511 & I $(0.00,0.39)$ & I & 0.020 \\
\hline | & $4-5-13$ & 1 & 0.093 & 1 & 0.171 & 1 & 0.546 & । & 0.585 & $(0.00,0.43)$ & । & 0.000 \\
\hline | & $1-4-8$ & 1 & 0.092 & । & 0.126 & 1 & 0.728 & । & 0.467 & $1(0.00,0.34)$ & I & 0.000 \\
\hline | & $2-3-13$ & 1 & 0.084 & । & 0.234 & | & 0.360 & | & 0.719 & I $(0.00,0.54)$ & | & 0.023 \\
\hline | & $1-2-6$ & I & 0.084 & I & 0.194 & | & 0.431 & I & 0.666 & $1(0.00,0.46)$ & I & 0.025 \\
\hline | & $2-4-8$ & 1 & 0.076 & 1 & 0.081 & 1 & 0.933 & । & 0.351 & $(0.00,0.24)$ & i & 0.000 \\
\hline | & $2-3-8$ & I & 0.075 & । & 0.089 & | & 0.838 & । & 0.402 & $1(0.00,0.25)$ & । & 0.000 \\
\hline | & $1-2-4$ & | & 0.070 & । & 0.108 & | & 0.651 & । & 0.515 & $1(0.00,0.28)$ & | & 0.009 \\
\hline | & $1-2-8$ & 1 & 0.067 & 1 & 0.223 & | & 0.300 & । & 0.764 & $1(0.00,0.50)$ & । & 0.028 \\
\hline i & $2-9-11$ & i & 0.067 & i & 0.139 & i & 0.481 & i & 0.631 & $(0.00,0.34)$ & | & 0.000 \\
\hline | & $1-3-8$ & | & 0.062 & | & 0.127 & | & 0.488 & | & 0.626 & $1(0.00,0.31)$ & I & 0.000 \\
\hline | & $1-3-4$ & | & 0.060 & | & 0.107 & 1 & 0.564 & | & 0.573 & I $(0.00,0.27)$ & | & 0.015 \\
\hline i & $3-4-5$ & i & 0.060 & i & 0.162 & i & 0.368 & i & 0.713 & $(0.00,0.38)$ & i & 0.039 \\
\hline | & $3-5-7$ & I & 0.056 & । & 0.307 & 1 & 0.182 & । & 0.855 & I $(0.00,0.66)$ & । & 0.048 \\
\hline | & $4-5-9$ & | & 0.052 & 1 & 0.148 & | & 0.353 & | & 0.724 & $1(0.00,0.34)$ & | & 0.000 \\
\hline | & $2-3-4$ & 1 & 0.051 & 1 & 0.106 & 1 & 0.485 & | & 0.628 & $\mid(0.00,0.26)$ & | & 0.018 \\
\hline i & $2-4-14$ & i & 0.049 & i & 0.130 & i & 0.377 & i & 0.706 & $(0.00,0.30)$ & i & 0.000 \\
\hline । & $1-5-9$ & I & 0.048 & । & 0.143 & | & 0.333 & । & 0.739 & $1(0.00,0.33)$ & । & 0.001 \\
\hline$x^{2}$ & $3-4-9$ & 1 & 0.045 & | & 0.163 & i & 0.274 & i & 0.784 & $(0.00,0.37)$ & i & 0.025 \\
\hline
\end{tabular}




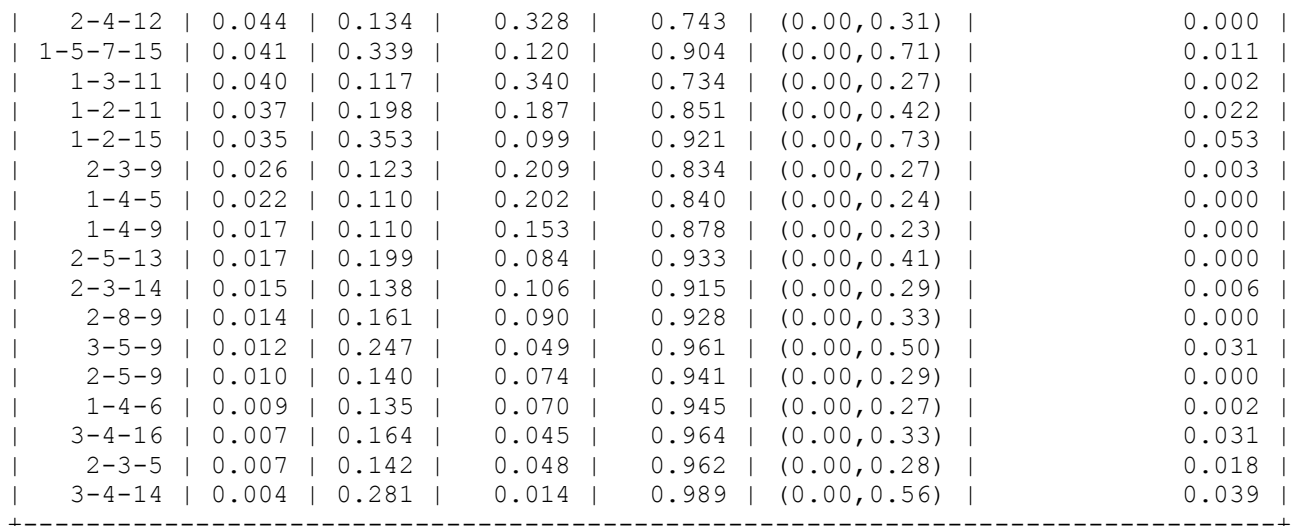

Table 7: Inconsistency factors - NMA with RCT and observational study

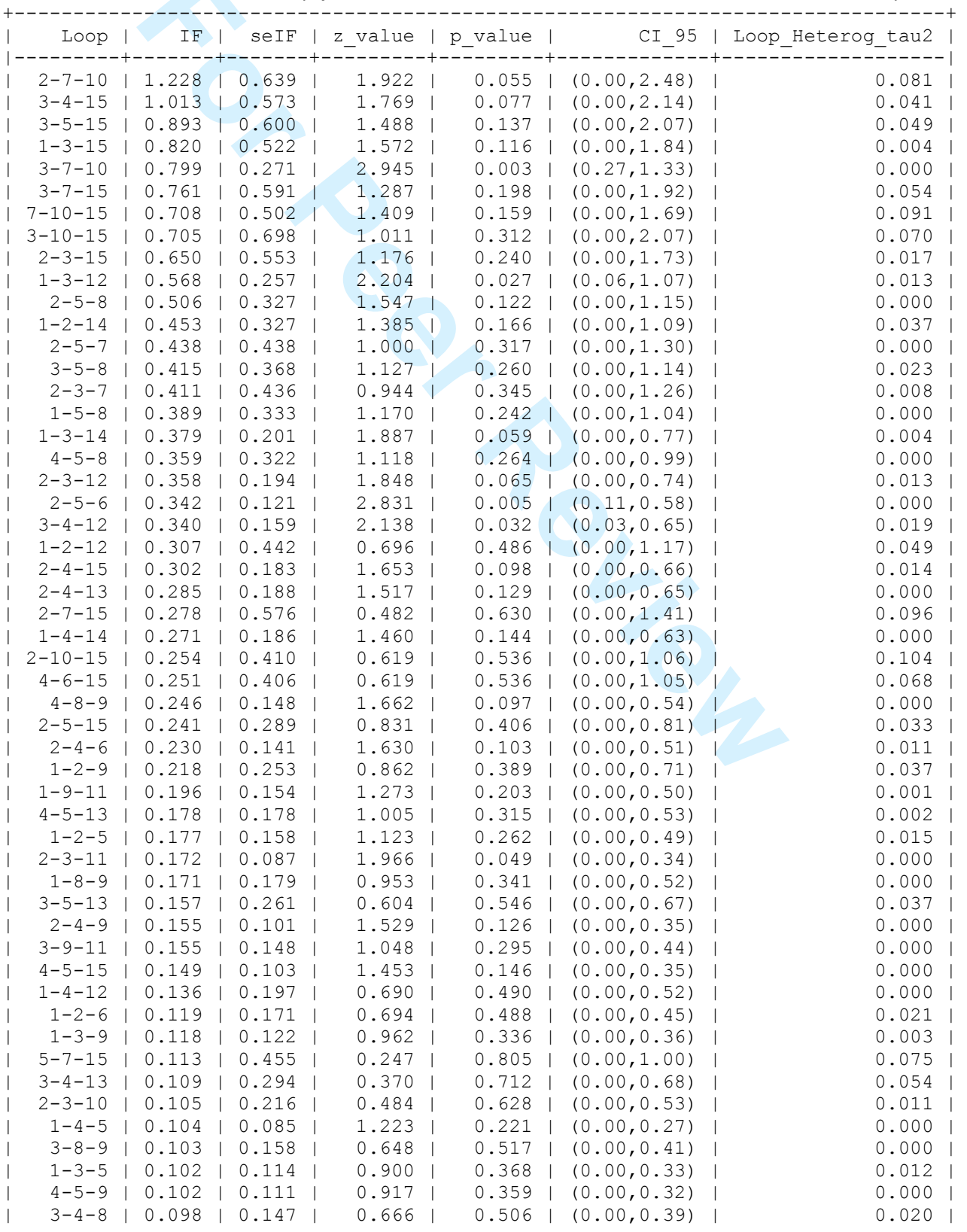




\begin{tabular}{l|c|l|}
0.427 & $(0.00,0.34)$ & 0.000 \\
0.294 & $(0.00,0.28)$ & 0.000 \\
0.393 & $(0.00,0.32)$ & 0.020 \\
0.365 & $(0.00,0.28)$ & 0.008 \\
0.713 & $(0.00,0.54)$ & 0.023 \\
0.411 & $(0.00,0.27)$ & 0.012 \\
0.769 & $(0.00,0.59)$ & 0.029 \\
0.350 & $(0.00,0.24)$ & 0.000 \\
0.407 & $(0.00,0.25)$ & 0.000 \\
0.764 & $(0.00,0.51)$ & 0.029 \\
0.631 & $(0.00,0.34)$ & 0.000 \\
0.445 & $(0.00,0.23)$ & 0.000 \\
0.623 & $(0.00,0.31)$ & 0.000 \\
0.538 & $(0.00,0.24)$ & 0.000 \\
0.626 & $(0.00,0.26)$ & 0.018 \\
0.849 & $(0.00,0.56)$ & 0.050 \\
0.707 & $(0.00,0.30)$ & 0.000 \\
0.877 & $(0.00,0.64)$ & 0.046 \\
0.785 & $(0.00,0.36)$ & 0.025 \\
0.743 & $(0.00,0.31)$ & 0.000 \\
0.723 & $(0.00,0.28)$ & 0.000 \\
0.733 & $(0.00,0.27)$ & 0.002 \\
0.859 & $(0.00,0.43)$ & 0.023 \\
0.757 & $(0.00,0.25)$ & 0.006 \\
0.734 & $(0.00,0.22)$ & 0.000 \\
0.832 & $(0.00,0.26)$ & 0.002 \\
0.861 & $(0.00,0.31)$ & 0.032 \\
0.848 & $(0.00,0.25)$ & 0.000 \\
0.935 & $(0.00,0.41)$ & 0.000 \\
0.927 & $(0.00,0.33)$ & 0.000 \\
0.923 & $(0.00,0.28)$ & 0.005 \\
0.882 & $(0.00,0.18)$ & 0.000 \\
0.960 & $(0.00,0.50)$ & 0.031 \\
0.942 & $(0.00,0.29)$ & 0.000 \\
0.958 & $(0.00,0.33)$ & 0.031 \\
0.970 & $(0.00,0.28)$ & 0.028 \\
0.992 & $(0.00,0.55)$ & \\
0.993 & $(0.00,0.48)$ & \\
\hline---1 & & \\
& &
\end{tabular}

Table 8: Estimates from the node-splitting approach for assessing inconsistency.

\begin{tabular}{|c|c|c|}
\hline \multirow[b]{2}{*}{ comparison split } & \multicolumn{2}{|c|}{$\begin{array}{c}\text { difference between direct and indirect evidence } \\
\text { (standard error) }\end{array}$} \\
\hline & only RCTs & RCTs and observational \\
\hline $1 \mathrm{v} 4$ & $0.03(0.08)$ & $0.05(0.07)$ \\
\hline $1 \mathrm{v} 5$ & $0.12(0.09)$ & $0.10(0.08)$ \\
\hline $1 \mathrm{v} 6$ & $0.07(0.13)$ & $0.08(0.10)$ \\
\hline $1 \mathrm{v} 15$ & $0.05(0.25)$ & $0.02(0.12)$ \\
\hline $4 \mathrm{v} 5$ & $0.09(0.10)$ & $0.02(0.08)$ \\
\hline $4 \mathrm{v} 6$ & $0.09(0.12)$ & $0.17(0.10)$ \\
\hline $4 \mathrm{v} 15$ & $0.37(0.23)$ & $0.25(0.12)$ \\
\hline $5 \mathrm{v} 6$ & - & $0.16(0.13)$ \\
\hline $5 v 15$ & - & $0.08(0.14)$ \\
\hline $6 \mathrm{v} 15$ & - & $0.11(0.15)$ \\
\hline
\end{tabular}

\subsection{Results: approach $B$}

The model estimates for the model parameters $\beta^{a g e}, \beta^{\text {dur }}$ and $\rho_{\text {age-dur }}$ are similar to the ones presented in Table 5 for all explored scenarios. The estimate for the heterogeneity standard deviation, $\tau$, does not change across the different scenarios: 
1

2

3

4

5

6

7

8

9

10

11

12

13

14

15

16

17

18

19

20

21

22

23

24

25

26

27

28

29

30

31

32

33

34

35

36

37

38

39

40

41

42

43

44

45

46

47

48

49

50

51

52

53

54

55

56

57

58

59

60
$0.080(0.042,0.120)$. This should be expected since in model B the NRSs are used to formulate informative prior distributions, and do not have any effect on the estimation of $\tau$. 


\section{OpenBUGS code for the presented models}

Here we provide the codes used to implement the Bayesian models presented in the main paper (used in the schizophrenia example). Comments on the code are written in blue. The dataset includes 167 RCTs and one non-randomized study. The following data are required as input:

ns: number of RCTs (in our example 167)

nt: number of treatments in the network (in our example 16)

agedur: a table with dimensions $n s \times 2$, with the mean patients' age and mean duration of illness for each study. These two covariates are standardized (by subtracting the mean and dividing by the standard deviation) so as to improve convergence

t: a table with dimensions $n s \times 4$, with information on the treatments being compared in each study. Each study is coded as a number between 1 and nt. A two-arm study has two entries in $t$ and the rest are set equal to NA. A three-arm study has three entries, etc. In our example the maximum number of arms in an RCT is 4.

na: a vector with the number of arms in each RCT (2 for two-armed studies, 3 for three-armed studies etc.)

ref: the code of the reference treatment of the network. The choice of treatment to be the reference is arbitrary.

regress.ref: the code of the treatment to be the reference in meta-regression. In our case we chose placebo.

$\mathrm{y}$ : a table with the mean outcome for each study arm, with dimensions $n s \times n t$. When a treatment is not included in a study the corresponding entry is set to NA.

sd: a table with the standard deviations for each study arm, with dimensions $n s \times n t$. When a treatment is not included in a study the corresponding entry is arbitrarily imputed with a non-negative number, e.g. set equal to zero.

$\mathrm{n}$ : a table with the number of patients randomized in each study arm, with dimensions $n s \times n t$. When a treatment is not included in a study the corresponding entry is set to one.

nt.NRS: the number of treatments in the non-randomized study. In our example 5 treatments are included.

tNRS: a vector with the treatments included in the non-randomized study. 
yobs: a vector with the relative treatment effect estimates from the observational study, for all other treatments vs the treatment indicated in tNRS[1]. In our example yobs has 4 elements.

s11, s22, s33, s44, s12, s13, s14, s23, s24, s34: the within-trial variance-covariance matrix corresponding to yobs. In our example this matrix is $4 \times 4$.

\subsection{Naïve analysis}

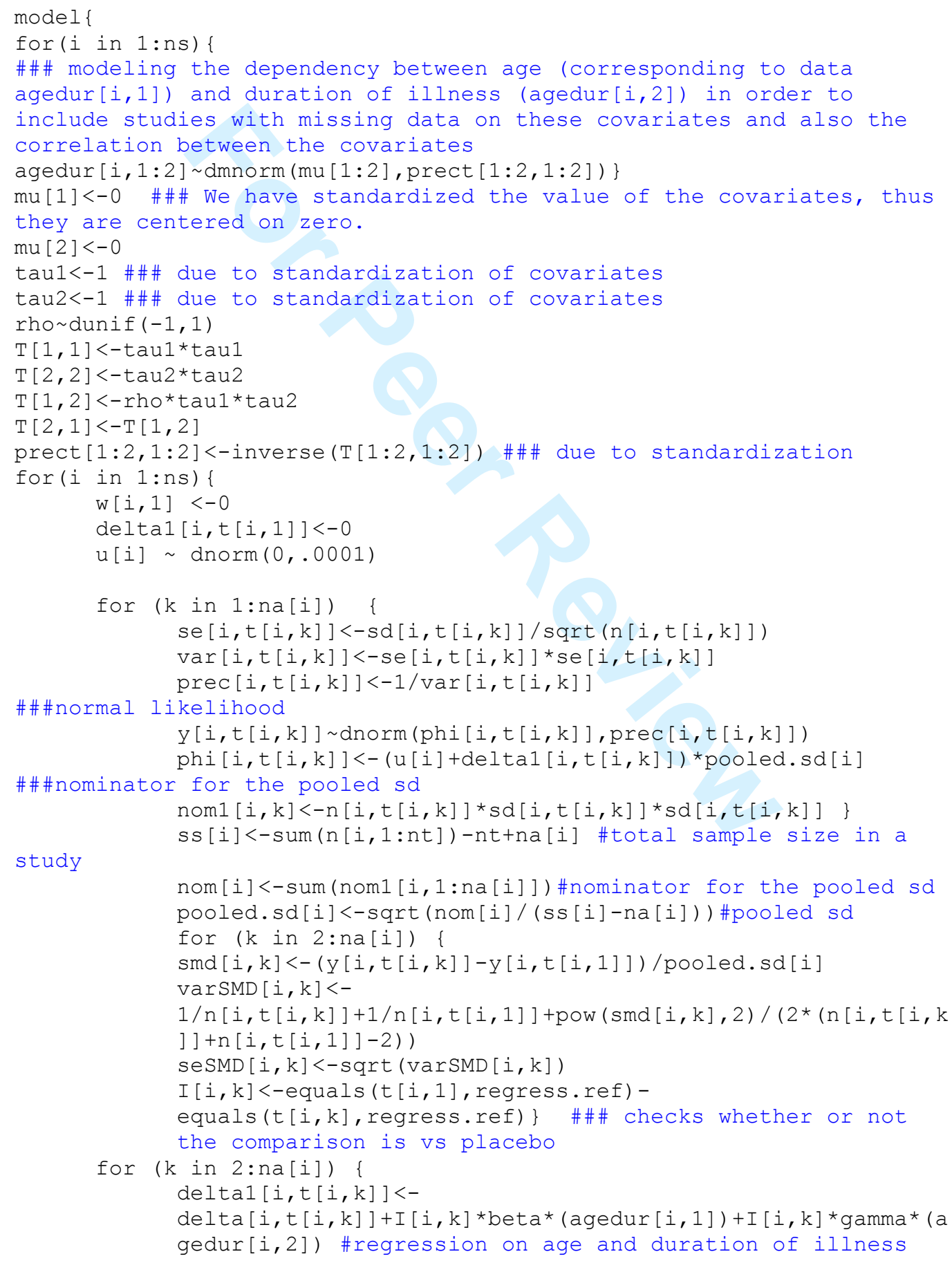


$\operatorname{delta}[i, t[i, k]] \sim \operatorname{dnorm}(\operatorname{md}[i, t[i, k]], \operatorname{PRECd}[i, t[i, k]])$

$\operatorname{md}[i, t[i, k]]<-d[t[i, k]]-d[t[i, 1]]+\operatorname{sw}[i, k]$

$\operatorname{PRECd}[i, t[i, k]]<-1 /(\operatorname{taud}[i, t[i, k]] * \operatorname{taud}[i, t[i, k]])$

taud $[i, t[i, k]]<-$ tau $* 2 *(k-1) / k$

$w[i, k]<-(\operatorname{delta}[i, t[i, k]]-d[t[i, k]]+d[t[i, 1]])$

\#adjustment, multi-arm RCTs

$\mathrm{sw}[\mathrm{i}, \mathrm{k}]<-\operatorname{sum}(\mathrm{w}[\mathrm{i}, 1: \mathrm{k}-1]) /(\mathrm{k}-1)\}\}$

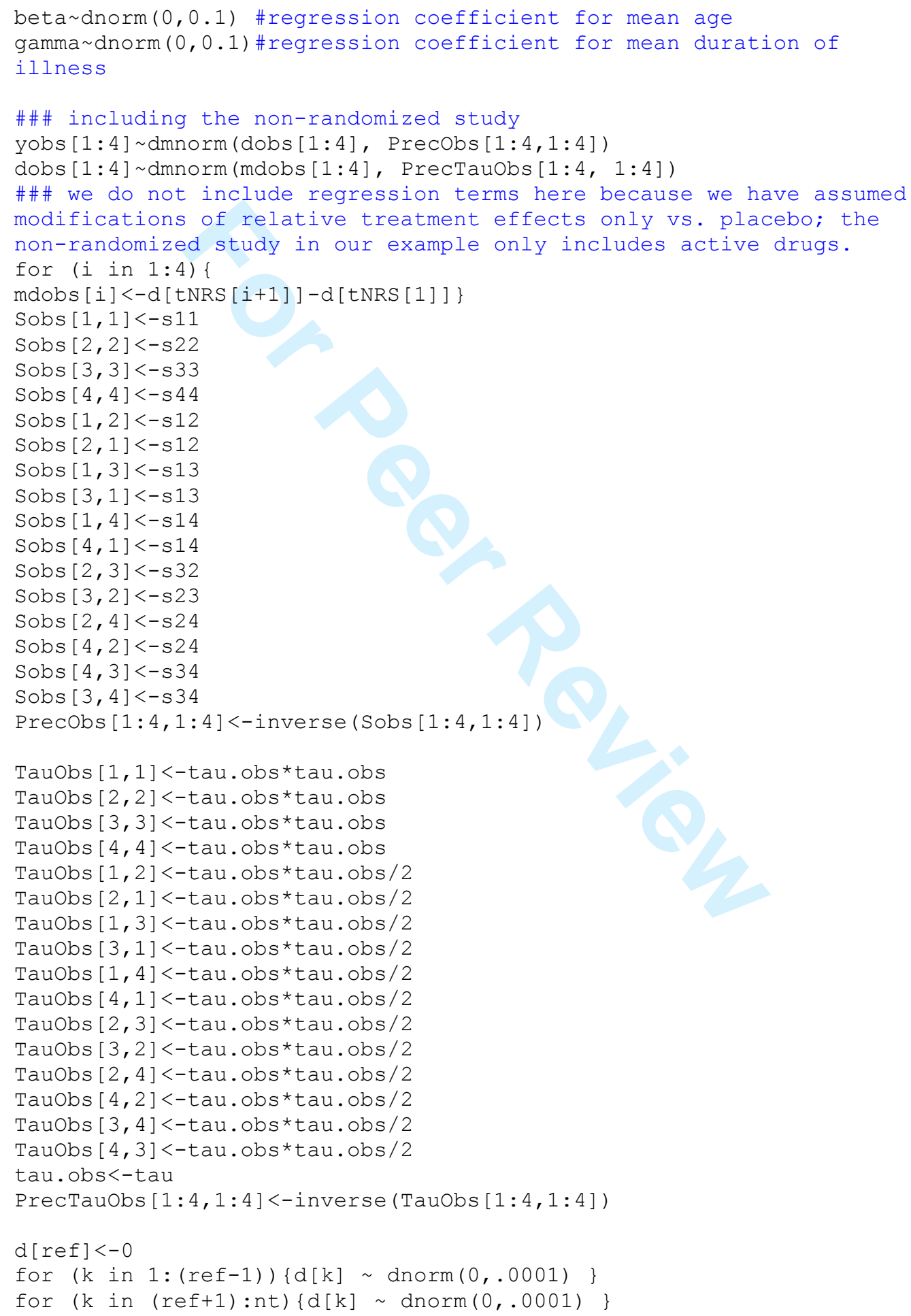




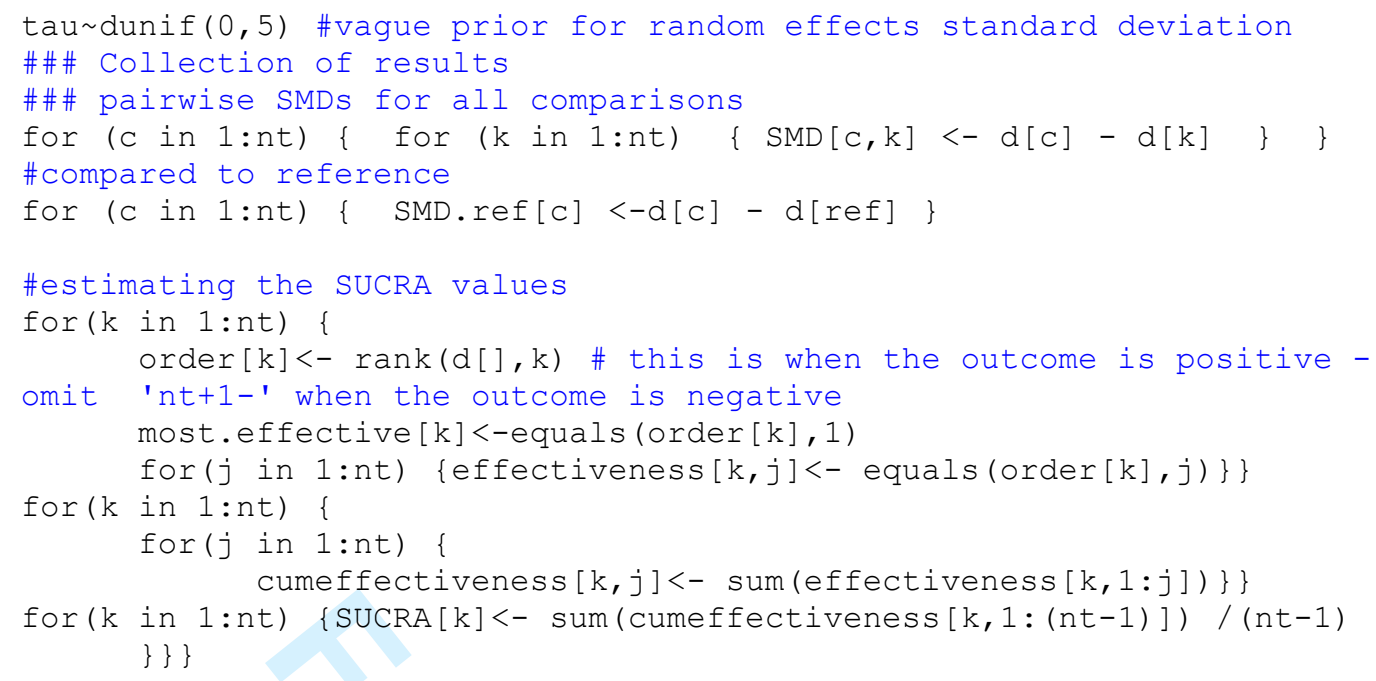

\subsection{Using non-randomized evidence as prior information}

model \{

for ( $i$ in $1:$ ns) \{

\#\#\# modeling the dependency between age (corresponding to data agedur[i,1]) and duration of illness (agedur[i,2]) in order to include studies with missing data on these covariates and also the correlation between the covariates agedur $[i, 1: 2] \sim \operatorname{dmnorm}(\mathrm{mu}[1: 2]$, prect $[1: 2,1: 2])\}$

mu[1]<-0 \#\#\# We have standardized the value of the covariates, thus they are centered on zero. 
$\operatorname{varSMD}[\mathrm{i}, \mathrm{k}]<-$

$1 / \mathrm{n}[\mathrm{i}, \mathrm{t}[\mathrm{i}, \mathrm{k}]]+1 / \mathrm{n}[\mathrm{i}, \mathrm{t}[\mathrm{i}, 1]]+\operatorname{pow}(\operatorname{smd}[\mathrm{i}, \mathrm{k}], 2) /(2 *(\mathrm{n}[\mathrm{i}, \mathrm{t}[\mathrm{i}, \mathrm{k}$

]$]+n[i, t[i, 1]]-2))$

$\operatorname{seSMD}[i, k]<-\operatorname{sqrt}(\operatorname{varSMD}[i, k])$

$I[i, k]<-e q u a l s(t[i, 1]$, regress.ref) -

equals (t $[i, k]$, regress.ref) $\}$ \#\#\# checks whether or not

the comparison is vs placebo

for ( $k$ in 2 :na [i]) \{

delta1 $[i, t[i, k]]<-$

delta $[i, t[i, k]]+I[i, k]$ *beta* (agedur $[i, 1])+I[i, k]$ *gamma * (a

gedur[i,2]) \#regression on age and duration of illness

delta $[i, t[i, k]] \sim \operatorname{dnorm}(\operatorname{ma}[i, t[i, k]], \operatorname{PRECd}[i, t[i, k]])$

$\operatorname{md}[i, t[i, k]]<-d[t[i, k]]-d[t[i, 1]]+s w[i, k]$

$\operatorname{PRECd}[i, t[i, k]]<-1 /(\operatorname{taud}[i, t[i, k]] * \operatorname{taud}[i, t[i, k]])$

taud $[i, t[i, k]]<-\operatorname{tau} * 2 *(k-1) / k$

$w[i, k]<-(\operatorname{delta}[i, t[i, k]]-d[t[i, k]]+d[t[i, 1]])$

\#adjustment, multi-arm RCTs

$\mathrm{sw}[\mathrm{i}, \mathrm{k}]<-\operatorname{sum}(\mathrm{w}[\mathrm{i}, 1: \mathrm{k}-1]) /(\mathrm{k}-1)\}\}$

beta dnorm $(0,0.1)$ \#regression coefficient for mean age

gamma dnorm $(0,0.1)$ \#regression coefficient for mean duration of

illness

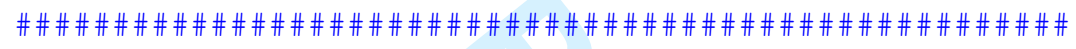

\#\#\# formulating informative priors based on the observational

estimates

yobs [1:4] dmnorm (d.obs [1:4], Precobs [1:4,1:4])

\#\#\# we do not include regression terms here because we have assumed modifications of relative treatment effects only vs. placebo; the non-randomized study in our example only includes active drugs.

Sobs $[1,1]<-s 11 / w w+t a u . n a i v e * t a u . n a i v e$ Sobs $[2,2]<-s 22 / w w+t a u . n a i v e * t a u . n a i v e$ Sobs $[3,3]<-s 33 / w w+t a u . n a i v e * t a u . n a i v e$ Sobs $[4,4]<-s 44 / w w+t a u . n a i v e * t a u . n a i v e$ Sobs $[1,2]<-s 12 /$ ww+tau. naive*tau.naive/2 Sobs $[2,1]<-s 12 / w w+t a u \cdot n a i v e * t a u \cdot n a i v e / 2$ Sobs $[1,3]<-s 13 / w w+t a u . n a i v e * t a u \cdot n a i v e / 2$ Sobs $[3,1]<-s 13 / w w+t a u \cdot n a i v e * t a u . n a i v e / 2$ Sobs $[1,4]<-s 14 /$ ww+tau. naive*tau.naive/2 Sobs $[4,1]<-s 14 / w w+t a u \cdot n a i v e * t a u \cdot n a i v e / 2$ Sobs $[2,3]<-s 32 / w w+t a u \cdot n a i v e * t a u \cdot n a i v e / 2$ Sobs $[3,2]<-s 23 / w w+t a u . n a i v e * t a u . n a i v e / 2$ Sobs $[2,4]<-s 24 / w w+t a u \cdot n a i v e * t a u . n a i v e / 2$ Sobs $[4,2]<-s 24 / w w+t a u . n a i v e * t a u . n a i v e / 2$ Sobs $[4,3]<-s 34 / w w+t a u \cdot n a i v e * t a u \cdot n a i v e / 2$ Sobs $[3,4]<-s 34 /$ ww+tau.naive*tau.naive/2 Precobs $[1: 4,1: 4]<-$ inverse (Sobs $[1: 4,1: 4]$ )

tau.naive $<-0.085$

ww dunif $(0,0.3)$ \#\#\# this is used to down-weight observational evidence

d.obs $[1] \sim \operatorname{dnorm}(0, .0001)$

d.obs [2] dnorm $(0, .0001)$

d.obs [3] dnorm $(0, .0001)$

d.obs [4] dnorm $(0, .0001)$

\#\#\# the relative effect estimates from the observational study. d [ 4 ] $<-d$.obs [1]

$d[5]<-d$.obs [2]

$d[6]<-d$.obs [3] 
$d[15]<-d$. obs $[4]$

$d[r e f]<-0$

for $(\mathrm{k}$ in $2: 3)\{\mathrm{d}[\mathrm{k}]$ dnorm $(0, .0001)\}$ \#\#\# here we assign

uninformative priors to the basic parameters not informed by the

observational evidence

for $(k$ in $7: 14)\{d[k] \sim \operatorname{dnorm}(0, .0001)\}$

$d[16] \sim \operatorname{dnorm}(0, .0001)$

tau dunif $(0,5)$ \#vague prior for random effects standard deviation

\#\#\# Collection of results

\#\#\# pairwise SMDs for all comparisons

for (c in 1:nt) \{ for (k in $1: n t) \quad\{\operatorname{SMD}[c, k]<-d[c]-d[k]\}$ \}

\#compared to reference

for (c in 1:nt) \{ SMD.ref[c] <-d[c] - d[ref] \}

\#estimating the SUCRA values

for (k in $1: n t) \quad\{$ order $[k]<-\operatorname{rank}(d[], k)$ \# this is when the outcome is positive -

omit 'nt+1-' when the outcome is negative most.effective $[k]<-$ equals (order $[k], 1$ ) for (j in $1: n t) \quad$ effectiveness $[k, j]<-$ equals (order $[k], j)\}\}$

for $(k$ in $1: n t) \quad\{$ for $(j$ in $1: n t) \quad\{$ cumeffectiveness $[k, j]<-\operatorname{sum}($ effectiveness $[k, 1: j])\}\}$

for (k in $1: n t) \quad\{$ SUCRA $[k]<-$ sum (cumeffectiveness $[k, 1:(n t-1)]) /(n t-1)$ \} \} \}

\subsection{Three-level hierarchical model (C1)}

Data required: as before, but now the number of studies per design need to be also included in the dataset, ns1, ns2, ns3, ns 4 etc.. Also the data from the studies in the structures $y, s d$, t, etc. need to be ordered per design. E.g. in y the first ns1 studies are of design 1, the next ns 2 studies are design 2, etc. In this example we have 4 designs (hig RoB RCTs, unclear RoB RCTs, low RoB RCTs and NRS)

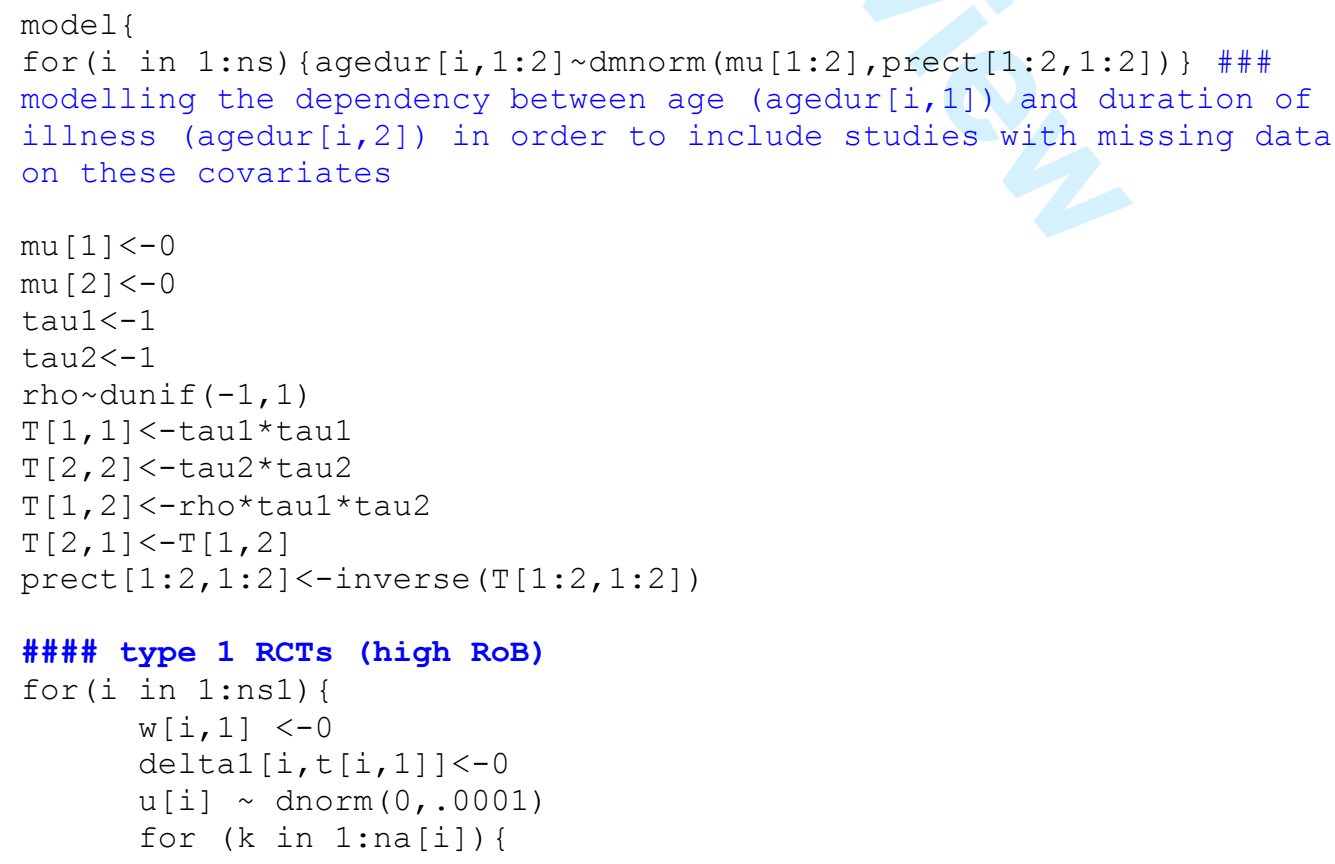


$\operatorname{se}[i, t[i, k]]<-\operatorname{sd}[i, t[i, k]] / \operatorname{sqrt}(n[i, t[i, k]])$

$\operatorname{var}[i, t[i, k]]<-\operatorname{se}[i, t[i, k]] \star \operatorname{se}[i, t[i, k]]$

$\operatorname{prec}[i, t[i, k]]<-1 / \operatorname{var}[i, t[i, k]]$

\#normal likelihood

$y[i, t[i, k]] \sim \operatorname{dnorm}(\operatorname{phi}[i, t[i, k]], \operatorname{prec}[i, t[i, k]])$

phi $[i, t[i, k]]<-(u[i]+\operatorname{deltal}[i, t[i, k]]) *$ pooled.sd $[i]$

\#calculate the pooled SD

nom $1[i, k]<-n[i, t[i, k]] * s d[i, t[i, k]] * s d[i, t[i, k]]$

\}\#nominator for the pooled sd

ss $[i]<-\operatorname{sum}(n[i, 1: n t])-n t+n a[i]$ \#total sample size in a study

nom[i]<-sum(nom1[i,1:na[i]]) \#nominator for the pooled sd

pooled.sd[i]<-sqrt(nom[i]/(ss[i]-na[i]))\# pooled sd

for (k in $2: n a[i]) \quad\{$

smd $[i, k]<-(y[i, t[i, k]]-y[i, t[i, 1]]) / \operatorname{pooled} . s d[i]$

$\operatorname{varSMD}[i, k]<-$

$1 / \mathrm{n}[\mathrm{i}, \mathrm{t}[\mathrm{i}, \mathrm{k}]]+1 / \mathrm{n}[\mathrm{i}, \mathrm{t}[\mathrm{i}, 1]]+\operatorname{pow}(\operatorname{smd}[\mathrm{i}, \mathrm{k}], 2) /(2 *(\mathrm{n}[\mathrm{i}$

$, t[i, k]]+n[i, t[i, 1]]-2))$

$\operatorname{seSMD}[i, k]<-\operatorname{sqrt}(\operatorname{varSMD}[i, k])$

$I[i, k]<-e q u a l s(t[i, 1], 3)$-equals (t $[i, k], 3) \quad \# \# \#$

checks whether or not the comparison is with placebo

\}

for (k in 2:na[i]) \{

deltal $[i, t[i, k]]<-\operatorname{delta}[i, t[i, k]]$

$+I[i, k] *$ beta* (agedur $[i, 1])+I[i, k] *$ gamma* (agedur $[i, 2])$ delta $[i, t[i, k]] \sim$

$\operatorname{dnorm}(\operatorname{md}[i, t[i, k]], \operatorname{taud} . I[i, t[i, k]])$

\#

trial-specific LOR distributions $\operatorname{md}[i, t[i, k]]<-\quad d R C T . I[t[i, k]]-\operatorname{dRCT} \cdot I[t[i, 1]]+$ $\mathrm{sw}[\mathrm{i}, \mathrm{k}]$

taud.I $[i, t[i, k]]<-\operatorname{tau} . I * 2 *(k-1) / k$

$\mathrm{w}[\mathrm{i}, \mathrm{k}]<-(\operatorname{delta}[\mathrm{i}, \mathrm{t}[\mathrm{i}, \mathrm{k}]]-\operatorname{dRCT} . \mathrm{I}[\mathrm{t}[\mathrm{i}, \mathrm{k}]]+$ dRCT.I[t[i,1]]) \#adjustment, multi-arm RCTs

$\mathrm{sw}[\mathrm{i}, \mathrm{k}]<-\operatorname{sum}(\mathrm{w}[\mathrm{i}, 1: \mathrm{k}-1]) /(\mathrm{k}-1) \quad$ \# cumulative

adjustment for multi-arm trials

\}

SD.I dnorm $(0,1) I(0$,$) \#vague prior for random effects standard$ deviation

tau. I $<-1 /(\mathrm{SD} \cdot \mathrm{I} * \mathrm{SD} \cdot \mathrm{I})$

\#\#\# type II RCTs (unclear RoB)

for (i in $(n s 1+1):(n s 1+n s 2))\{$

$w[i, 1]<-0$

delta1 $[i, t[i, 1]]<-0$

$u[i] \sim \operatorname{dnorm}(0, .0001)$

for ( $k$ in 1 :na $[i]) \quad$ (

$\operatorname{se}[i, t[i, k]]<-\operatorname{sd}[i, t[i, k]] / \operatorname{sqrt}(n[i, t[i, k]])$

$\operatorname{var}[i, t[i, k]]<-\operatorname{se}[i, t[i, k]] \star \operatorname{se}[i, t[i, k]]$

$\operatorname{prec}[i, t[i, k]]<-1 / \operatorname{var}[i, t[i, k]]$

\#normal likelihood

$y[i, t[i, k]] \sim \operatorname{dnorm}(\operatorname{phi}[i, t[i, k]], \operatorname{prec}[i, t[i, k]])$

phi $[i, t[i, k]]<-(u[i]+\operatorname{deltal}[i, t[i, k]]) *$ pooled.sd [i]

\#calculate the pooled SD

$\operatorname{nom} 1[i, k]<-n[i, t[i, k]] * s d[i, t[i, k]] * s d[i, t[i, k]]$

\#nominator for the pooled sd 
$\operatorname{varSMD}[\mathrm{i}, \mathrm{k}]<-$

$1 / \mathrm{n}[\mathrm{i}, \mathrm{t}[\mathrm{i}, \mathrm{k}]]+1 / \mathrm{n}[\mathrm{i}, \mathrm{t}[\mathrm{i}, 1]]+\operatorname{pow}(\operatorname{smd}[\mathrm{i}, \mathrm{k}], 2) /(2 *(\mathrm{n}[\mathrm{i}, \mathrm{t}[\mathrm{i}, \mathrm{k}$ ]$+n[i, t[i, 1]]-2))$

\section{\#\#\#\# NRS}

y.NRS [1:4] dmnorm(theta.NRS [1:4], Prec.NRS [1:4,1:4]) \#\#\# likelihood of the single NRS study

theta.NRS [1:4] dmnorm(eff.NRS [1:4], Prec.T.NRS $[1: 4,1: 4])$ \#\#\# Random effects

eff.NRS [1]<-d.NRS [4]

eff.NRS [2]<-d.NRS [5]

eff.NRS [3]<-d.NRS [6]

eff.NRS [4]<-d.NRS [15]

ww $<-1$ \#\#\# variance inflation factor

S.NRS $[1,1]<-s 11 / W W$

S.NRS $[2,2]<-s 22 / w W$

S.NRS $[3,3]<-s 33 / w W$

S.NRS $[4,4]<-s 44 /$ WW 


\section{Page 57 of 58}

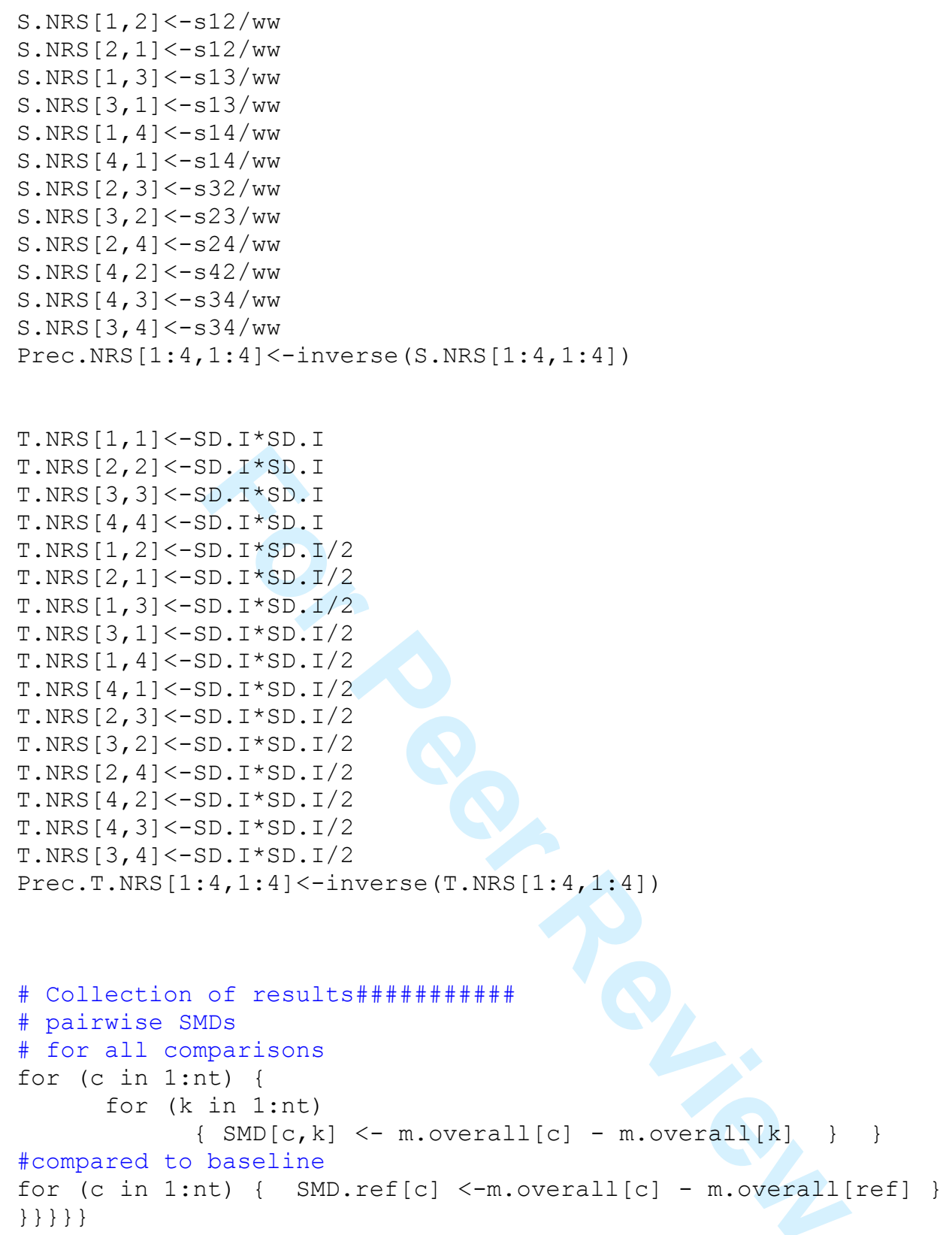




\section{References}

1. Cooper NJ, Sutton AJ, Morris D, Ades AE, Welton NJ: Addressing between-study heterogeneity and inconsistency in mixed treatment comparisons: Application to stroke prevention treatments in individuals with non-rheumatic atrial fibrillation. Statistics in Medicine 2009, 28:1861-1881.

2. Salanti G, Marinho V, Higgins JP: A case study of multiple-treatments metaanalysis demonstrates that covariates should be considered. JClinEpidemiol 2009, 62:857-864.

3. Efthimiou O, Debray TPA, van Valkenhoef G, Trelle S, Panayidou K, Moons KGM, Reitsma JB, Shang A, Salanti G, GetReal Methods Review Group: GetReal in network meta-analysis: a review of the methodology. Research Synthesis Methods 2016.

4. Dias S, Welton NJ, Sutton AJ, Caldwell DM, Lu G, Ades AE: Evidence synthesis for decision making 4: inconsistency in networks of evidence based on randomized controlled trials. MedDecisMaking 2013, 33:641-656.

5. Almalla M, Schröder JW, Pross V, Marx N, Hoffmann R: Three-year follow-up after treatment of bare-metal stent restenosis with first-generation or secondgeneration drug-eluting stents. Coron Artery Dis 2013, 24:165-170.

6. Almalla M, Schröder J, Pross V, Marx N, Hoffmann R: Paclitaxel-eluting balloon versus everolimus-eluting stent for treatment of drug-eluting stent restenosis. Catheterization and Cardiovascular Interventions 2014, 83:881-887.

7. Markovic S, Paliskyte R, Rottbauer W, Wöhrle J: Everolimus-eluting stents compared with paclitaxel-eluting stents for treatment of coronary in-stent restenoses. Cardiovasc Revasc Med 2012, 13:307-310.

8. Airoldi F, Briguori C, Iakovou I, Stankovic G, Biondi-Zoccai G, Carlino M, Chieffo A, Montorfano M, Cosgrave J, Michev I, Rogacka R, Sangiorgi GM, Colombo A: Comparison of sirolimus versus paclitaxel eluting stents for treatment of coronary in-stent restenosis. Am J Cardiol 2006, 97:1182-1187.

9. Ota H, Mahmoudi M, Kitabata H, Torguson R, Chen F, Satler LF, Suddath WO, Pichard AD, Waksman R: Safety and efficacy of limus-eluting stents and balloon angioplasty for sirolimus-eluting in-stent restenosis. Cardiovascular Revascularization Medicine 2015, 16:84-89.

10. Appleby CE, Khattar RS, Morgan K, Clarke B, Curzen N, Neyses L, FathOrdoubadi F: Drug eluting stents for the treatment of bare metal in-stent restenosis: long-term outcomes in real world practice. EuroIntervention 2011, 6:748-753.

11. Leucht S, Cipriani A, Spineli L, Mavridis D, Orey D, Richter F, Samara M, Barbui C, Engel RR, Geddes JR, Kissling W, Stapf MP, Lässig B, Salanti G, Davis JM: Comparative efficacy and tolerability of 15 antipsychotic drugs in schizophrenia: a multiple-treatments meta-analysis. Lancet 2013, 382:951-962. 